Revue scientifique sur la conception et l'aménagement de l'espace

\title{
L'Observatoire photographique national du paysage : transformations d'un modèle et hypothèses renouvelées de paysage
}

Photographic Observatory of the Landscape): Transformations of a Model and Renewed Hypotheses of the Landscape

\section{Frédérique Mocquet}

\section{(2) OpenEdition}

\section{Journals}

Édition électronique

URL : http://journals.openedition.org/paysage/7099

DOI : 10.4000/paysage.7099

ISSN : 1969-6124

Éditeur :

École nationale supérieure du paysage de Versailles-Marseille, Institut national des sciences appliquées Centre Val de Loire - École de la nature et du paysage, École nationale supérieure d'architecture et de paysage de Bordeaux, École nationale supérieure d'architecture et de paysage de Lille, Agrocampus Angers

\section{Référence électronique}

Frédérique Mocquet, «L'Observatoire photographique national du paysage : transformations d'un modèle et hypothèses renouvelées de paysage », Projets de paysage [En ligne], 15 | 2016, mis en ligne le 31 décembre 2016, consulté le 13 juin 2020. URL : http://journals.openedition.org/paysage/7099; DOI : https://doi.org/10.4000/paysage.7099

Ce document a été généré automatiquement le 13 juin 2020

Projets de paysage 


\title{
L'Observatoire photographique
} national du paysage : transformations d'un modèle et hypothèses renouvelées de paysage

\author{
Photographic Observatory of the Landscape): Transformations of a Model and
} Renewed Hypotheses of the Landscape

\section{Frédérique Mocquet}

\section{NOTE DE L'ÉDITEUR}

Un entretien donnant la parole aux photographes a été mené dans le cadre de la préparation de cet article. Vous pouvez lire ce texte « Regards artistiques sur l'observation photographique. Entretien avec Geoffroy Mathieu et Bertrand Stofleth, auteurs de "Paysages usagés, Observatoire photographique du paysage depuis le GR2013" » dans la rubrique « Matières premières » de ce même numéro (https:// journals.openedition.org/paysage/7186).

1 La visibilité du paysage est aujourd'hui extrême : des discours grand public aux champs spécialisés, le mot et l'image sont partout. Michael Jakob parle d'« omnipaysage " (Jakob, 2008, p. 7) pour exprimer la puissance de ce phénomène qu'il analyse comme le signe d'une inquiétude fondamentale des sociétés contemporaines. Bien que la politique $d u$ et par le paysage soit une réalité depuis le XVIII ${ }^{e}$ siècle, la notion est entrée dans le droit depuis peu. En France, la loi paysage du 8 janvier 1993 a donné une impulsion décisive, renforcée en 2006 par la ratification de la Convention européenne du paysage ${ }^{1}$. En officialisant une définition, ce texte opère un changement politique par une évolution, d'abord, des modèles. Il articule le passage d'une idéologie élitiste et protectionniste à une appréhension démocratique et évolutive incluant notamment les paysages dits "ordinaires». Il esquisse une vision holiste du paysage en tant que 
produit de la perception et combinaison d'éléments changeants, naturels et culturels, matériels et immatériels, à considérer simultanément. Cet énoncé, simple voire minimaliste, ne relève pas d'une intention de définir ce concept complexe mais est plutôt motivé par le désir de fonder les bases d'une réflexion commune. Pensé comme réalité, concept et discours, le paysage est progressivement envisagé comme vecteur pour atteindre, moyen pour penser, média pour communiquer des problématiques environnementales, urbanistiques, infrastructurelles, sociales, etc. Pierre Donadieu, géographe, synthétise la richesse préopérationnelle de ce concept : « Le paysage est une notion relationnelle, un entre-deux qui crée le sens des étendues et des lieux visibles. C'est pourquoi il se présente comme un outil de passage entre le visible et l'invisible, entre l'objectif et le subjectif, entre la science et l'art, entre l'écologique et le symbolique, entre les espaces vus et vécus, entre le matériel et le spirituel, entre la totalité et les parties, et surtout entre la connaissance et l'action " (Donadieu, 2007, p. 9).

2 Le paysage est donc envisagé comme un objet riche nourri par divers courants de pensées: géographique, écologique, philosophique, paysagiste, etc. Plus celui-ci est reconnu dans sa pluralité, plus sa portée s'enrichit, mais plus aussi ses fondations se fragilisent et sa manipulation se complexifie. Ainsi, la production d'une politique du paysage et des outils associés exige-t-elle une prise de position conceptuelle par une mise en œuvre transversale, dont l'enjeu réside dans la prise en compte de la dimension sensible et historico-culturelle autant que matérielle des réalités paysagères. C'est au début de ce processus d'outillage conceptuel, législatif et méthodologique appliqué à l'aménagement qu'est annoncé le 22 novembre 1989 l'Observatoire photographique national du paysage (OPNP). Jean Cabanel, chef de la nouvelle Mission du paysage rattachée au ministère de l'Environnement, présente en conseil des ministres son objectif de "constituer un fonds de séries photographiques qui permettent d'analyser les mécanismes et facteurs de transformation des espaces ainsi que les rôles des différents acteurs qui en sont la cause de façon à orienter favorablement l'évolution du paysage ». Le projet est conçu de 1991 à 1996 par Caroline Mollie-Stefulesco, paysagiste chargée de mission, assistée de Véronique Ristelhueber, documentaliste, et Daniel Quesney, photographe chargé du suivi artistique et technique. C'est un travail de terrain présentant un caractère fortement expérimental et itératif. Il est mené avec des Parcs naturels régionaux (Pilat, Livradois-Forez, Haute Vallée de Chevreuse, Armorique et Forêt d'Orient) qui contribuent à la définition des objectifs autant que de la méthode. 
Figure 1. Condrieu (69), vue sur le Rhône et abords (01000101), octobre 1992

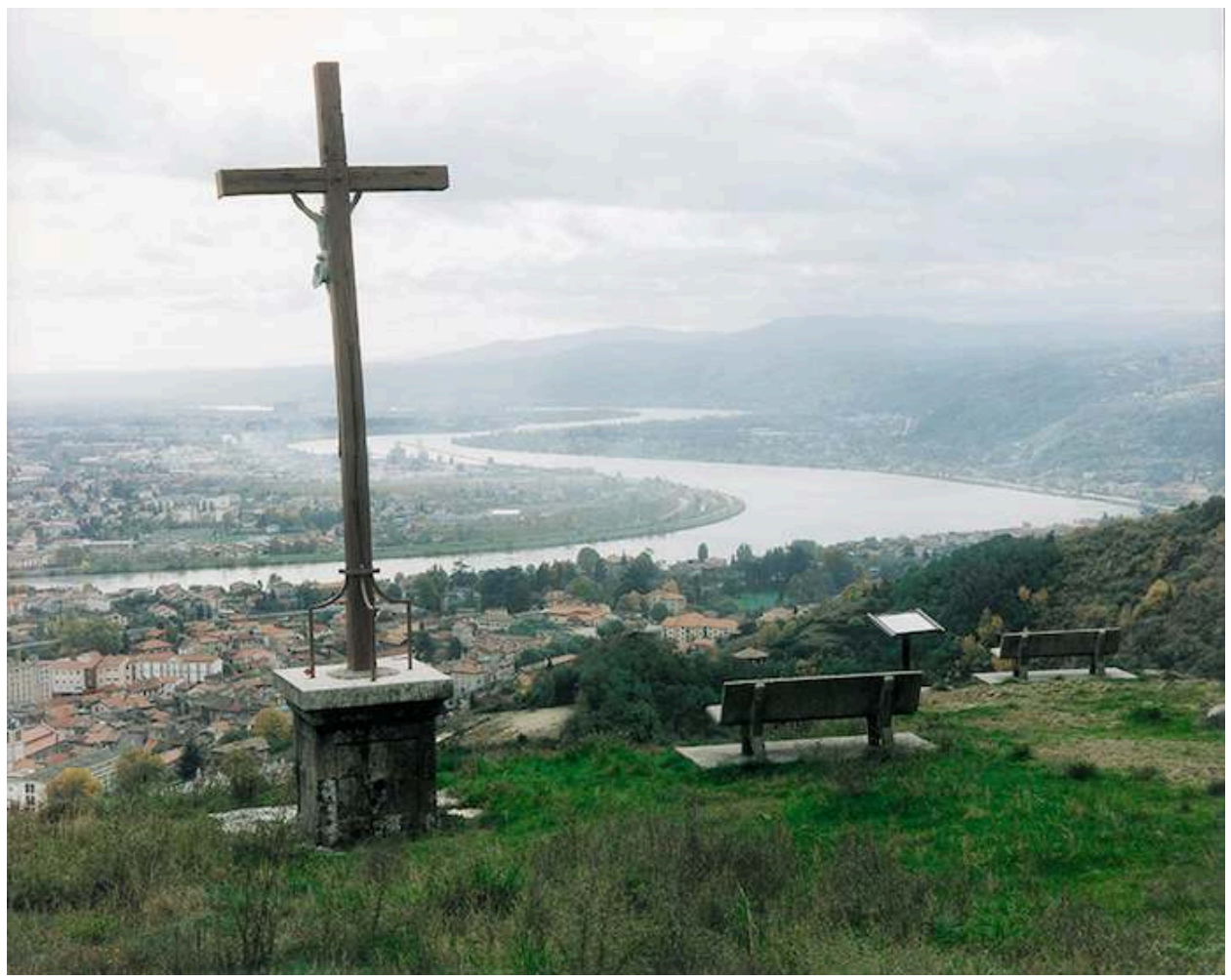

Itinéraire $n^{\circ} 1$, Parc naturel régional du Pilat.

L'itinéraire du Parc naturel régional du Pilat a été monté parmi les tout premiers, avec la photographe Sophie Riestelhueber (qui avait participé à la Mission photographique de la Datar). Sa proposition de travailler sur « les industries de la nature » a correspondu avec les préoccupations du parc, portant notamment sur la déprise agricole et les évolutions urbaines (explosions périurbaines, zones d'activités, affichages publicitaires, etc.)

Source : Sophie Ristelhueber, OPNP. 
Figure 2. Route de Saint-Étienne au Guizay - Au fond, Saint-Étienne, (01 0010 01), octobre 1992

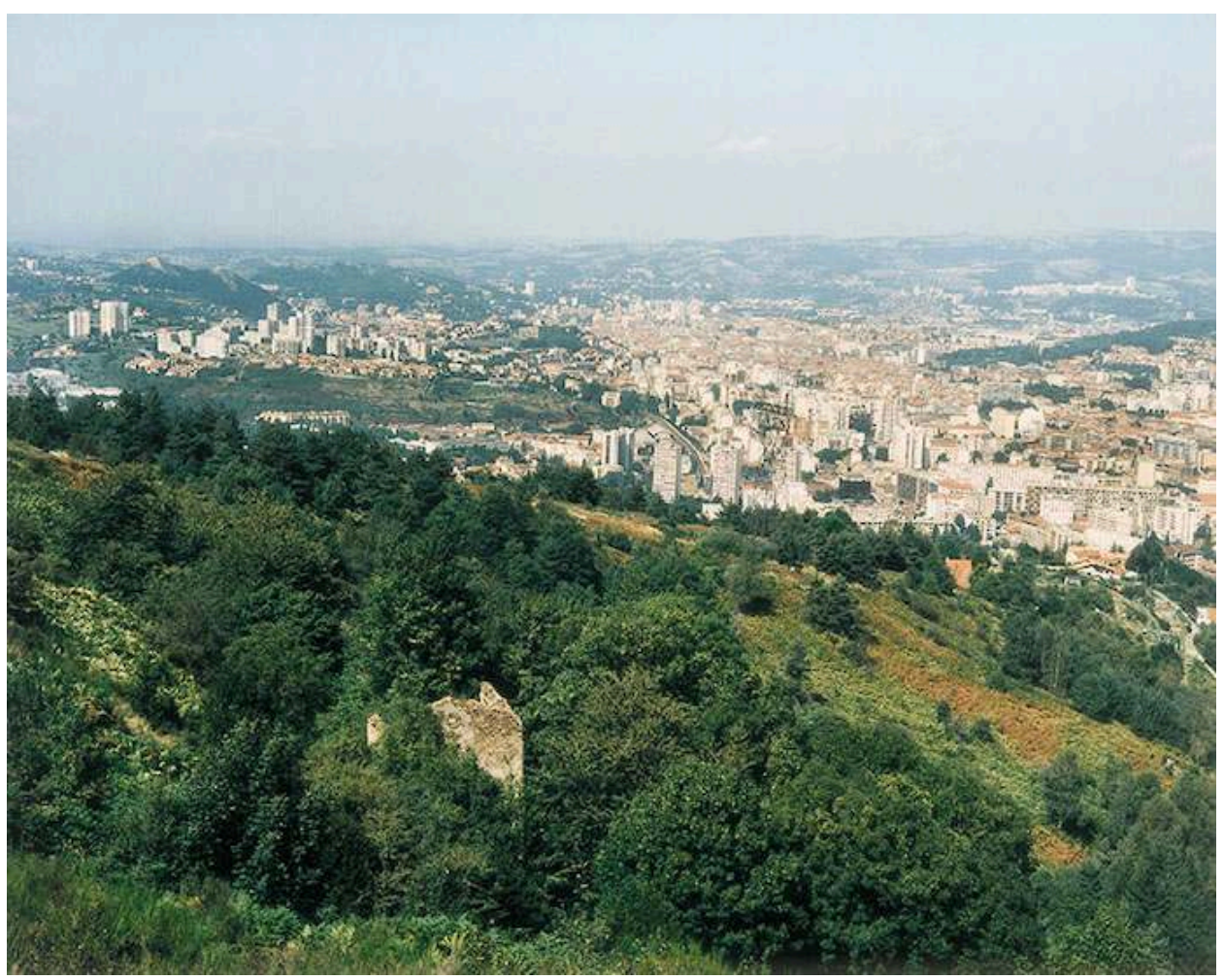

Itinéraire $n^{\circ} 1$, Parc naturel régional du Pilat.

Source : Sophie Ristelhueber, OPNP.

3 L'observatoire a jusqu'en 2008 une dimension nationale effective, puisqu'il fonctionne sous l'égide du Bureau des paysages. Vingt itinéraires dits « nationaux » sont montés en partenariat tripartite entre le ministère, une collectivité locale et un photographe. Après l'arrêt du financement et de l'encadrement pratique, le soutien du ministère devient symbolique. La démarche est érigée en modèle, dans le cadre d'initiatives indépendantes: on parle d'observatoires photographiques du paysage (OPP) pour désigner ces observatoires dits « locaux » (DGALN/DHUP, 2015). La dynamique est forte depuis l'orée des années 2000 et on répertorie une centaine de démarches héritières du programme national, revendiquant d'ailleurs toutes une filiation, qu'elles reprennent ou transforment la méthode. Mais ce succès est en fait paradoxal. En effet, on constate la multiplication des OPP tout en notant également la récurrence des réserves sur la « méthode ». Des « points noirs » sont énoncés, par exemple le caractère chronophage et le coût du montage et du maintien de l'itinéraire ainsi que certains aspects de la méthode perçus comme lourds et laborieux (l'appel à un photographe professionnel et le comité de pilotage par exemple). La complexité, qu'elle soit ressentie ou réelle, s'accompagne de difficultés d'utilisation de l'objet selon les objectifs annoncés : analyse des évolutions paysagères, évaluation des politiques publiques ou encore aide à la décision (Meeddat, 2008). Les porteurs sont souvent démunis quant à la manière de procéder, tant pour construire «l'outil » que pour l'utiliser et obtenir des « résultats » identifiables. Compte tenu des critiques et des difficultés récurrentes, pourquoi le modèle continue-t-il de séduire les acteurs et organismes en charge des territoires et des paysages? 
4 Un retour préalable sur le contexte et les hypothèses originelles permet d'abord de saisir « l'esprit » de l'OPNP et de comprendre l'attrait que la démarche qu'il a initiée suscite. Cet état des lieux met en lumière les originalités, spécificités et perspectives de ce modèle, permettant de le questionner comme projet paysager et projet photographique, projet de paysage par la photographie. Une analyse des fondements théoriques, conceptuels et formels est opérée en quelques points, confrontés ensuite à la mise en œuvre; cela afin d'observer l'écart supposé entre la rhétorique, partie prenante du projet, et le terrain. Des questions se posent, par exemple: «l'héritage OPNP » serait-il la reconduction à la fois des apports d'un programme expérimental, mais aussi de ses ambivalences, de ses lacunes, voire de ses contresens? Aussi, dans quelle mesure y aurait-il continuation de fragilités initiales et/ou transformation de principes modifiant les enjeux, objectifs, applications et intelligences du projet? Cette analyse historique et critique ciblée est le socle pour l'interrogation, ensuite, de la pertinence actuelle de la démarche, en regard des pensées des paysages de besoins et d'objectifs qui depuis 25 ans ont évolué. Enfin, une initiative particulière offre un support de réflexion sur les potentiels et perspectives de réactualisation de l'héritage. "L'Observatoire photographique des paysages depuis le GR2013 ", interrogation en actes du paysage contemporain et des possibilités de l'observation photographique appliquée aux territoires, permet en effet d'ouvrir la réflexion sur le paysage comme enjeu, ressource, outil, selon une acception aujourd'hui élargie et hybride du projet de territoire.

\section{Contextes}

5 L'OPNP est esquissé dans les années 1980, période de changement de paradigme spatial, culturel et politique. Caroline Mollie-Stefulesco se souvient $d$ '« une forte prise de conscience chez les professionnels de l'anarchie totale générée par les Trente Glorieuses et des retombées de l'agriculture industrielle à laquelle devait répondre une sortie progressive de la "politique d'aménagement planificatrice et verticale" qui prendrait en compte le paysage ${ }^{2} »$. Elle évoque également la découverte de la Mission photographique de la Datar comme un « choc émotionnel ». En effet, la Mission propose une rhétorique iconographique et textuelle qui montre les conséquences des politiques en cours et articule un changement de paradigme paysager global touchant les pratiques d'aménagement ainsi que la pensée et la représentation du paysage. Enfin, elle matérialise la concordance entre une politique culturelle et une politique spatiale, chacune en mutation. Dans le même temps, se pose la question de « la mort du paysage ${ }^{3}$ ». Le paysage n'est pas mort mais le public, l'usager, l'habitant ne semblent plus le reconnaître, s'y reconnaître. La crise n'est pas, en réalité, dans le fait observé, mais dans le décalage entre l'idée que l'on se fait individuellement et collectivement du paysage et le paysage «réel». Il s'agit donc d'une crise plus générale : des modèles conceptuels, d'action et de représentations. (Lenclud, 1995).»

6 C'est dans ce contexte d'effervescence et de remise en question que le projet émerge au sein de la Mission du paysage, qui débat alors des données susceptibles de fonder des politiques publiques. On constate le manque de moyens d'appréciation de l'état des paysages et de leurs changements, du point de vue de l'expérience vécue et on souhaite compléter les outils d'analyse quantitative avec des outils d'analyse qualitative, selon deux facteurs nouveaux : les perceptions et les effets du temps. Le 16 mars 1989, Jean 
Cabanel rédige une note d'intention qui envisage ces problématiques et les nouveaux besoins qu'elles engendrent. Ce premier texte, document de travail non publié, esquisse les enjeux fondateurs. On peut y lire: "Le paysage constitue un système de lecture visuelle de l'état de l'environnement ${ }^{4}$ ayant une connotation sensible. Le paysage est aussi un vecteur médiatique; en agissant sur certains des signes qui entrent dans sa composition il est possible de modifier les comportements et les schèmes de décision. Le paysage est donc un concept complexe dans lequel la composante sensible, c'est-à-dire subjective, est essentielle. Compte tenu de cette complexité et de son caractère immatériel, l'évolution ne peut guère être saisie à travers des statistiques. Il faut faire appel à un ensemble de moyens de nature différente.» Des «campagnes de relevés photographiques et phytosociologiques" sont envisagées "pour saisir les évolutions visuelles, le moyen le plus précis et le plus économique consiste à effectuer des relevés photographiques. Ceux-ci seraient effectués par des photographes de haut niveau sur un ensemble d'itinéraires représentatifs de milieux naturels, agricoles, périurbains. Sur ces itinéraires les photographes auraient carte blanche pour saisir à travers leur objectif la réalité qu'ils ressentent, mais ils seraient également tenus d'effectuer des prises de vue parfaitement repérées et cadrées, effectuées à la même époque pour que puissent être appréciées les évolutions ».

Figure 3. Carvin - Lotissement et terril de Carvin (04 024 01), juin 1993

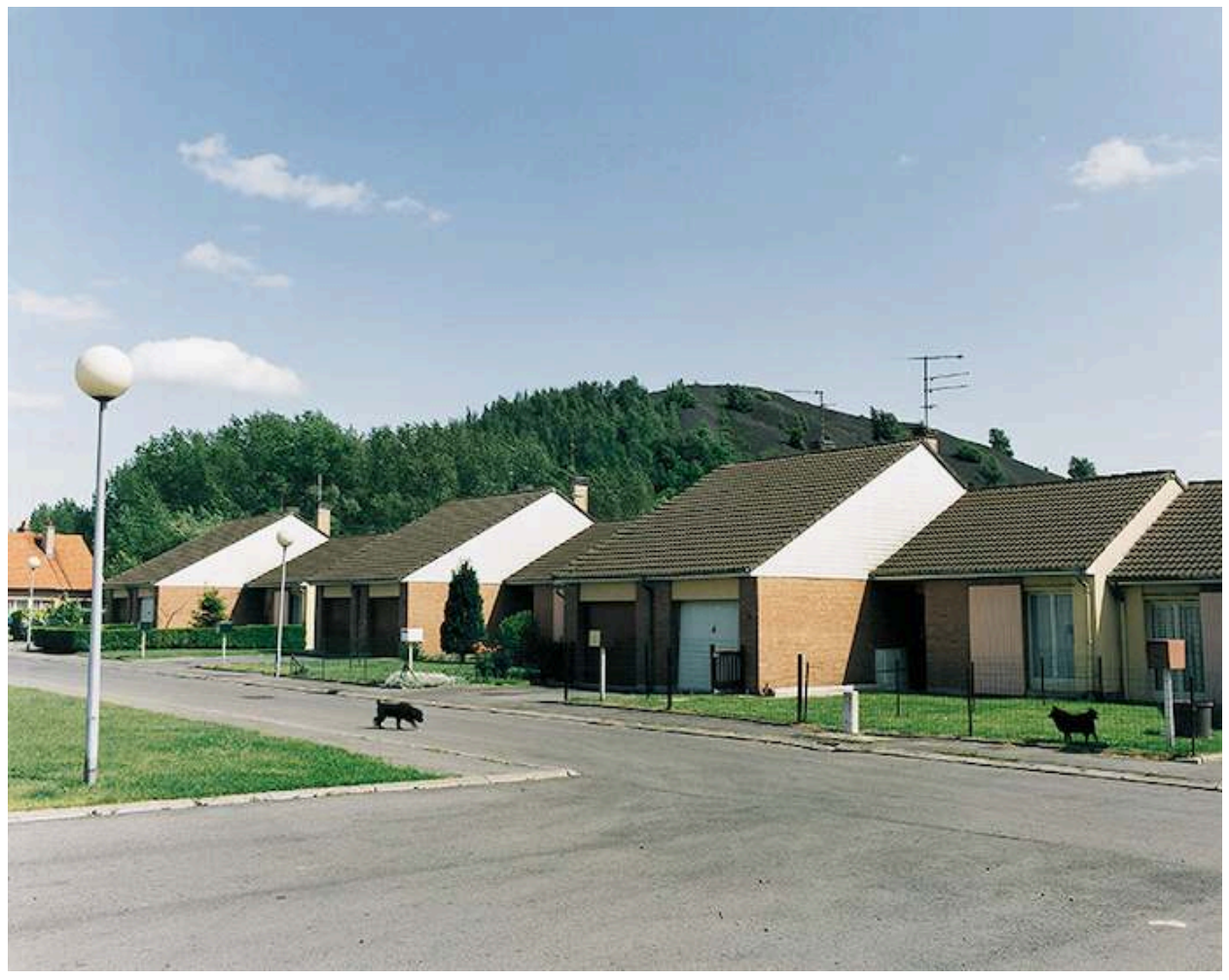

Itinéraire $n^{\circ} 4$, Nord-Pas-de-Calais (Dreal).

Cet itinéraire montre les différents types de milieux énoncés par Jean Cabanel dans sa première note d'intention : milieux naturels, agricoles, périurbains. S'articulant autour du projet de l'autoroute A1 et du TGV Arras/Lille, il permet d'aborder des problématiques à l'œuvre au nord de la France : grandes infrastructures (autoroute, TGV), centres commerciaux, zones d'activités et de loisirs, agriculture périurbaine, périphérie des grandes villes et entrées de ville, affichage et publicité, évolution de la végétation sur les terrils; et plus largement, d'illustrer des phénomènes présents sur tout le territoire français.

Source : Dominique Auerbacher, OPNP. 
Figure 4. Seclin - TGV et D925 passant sur voie ferrée et route en construction (04 034 01), juin 1993

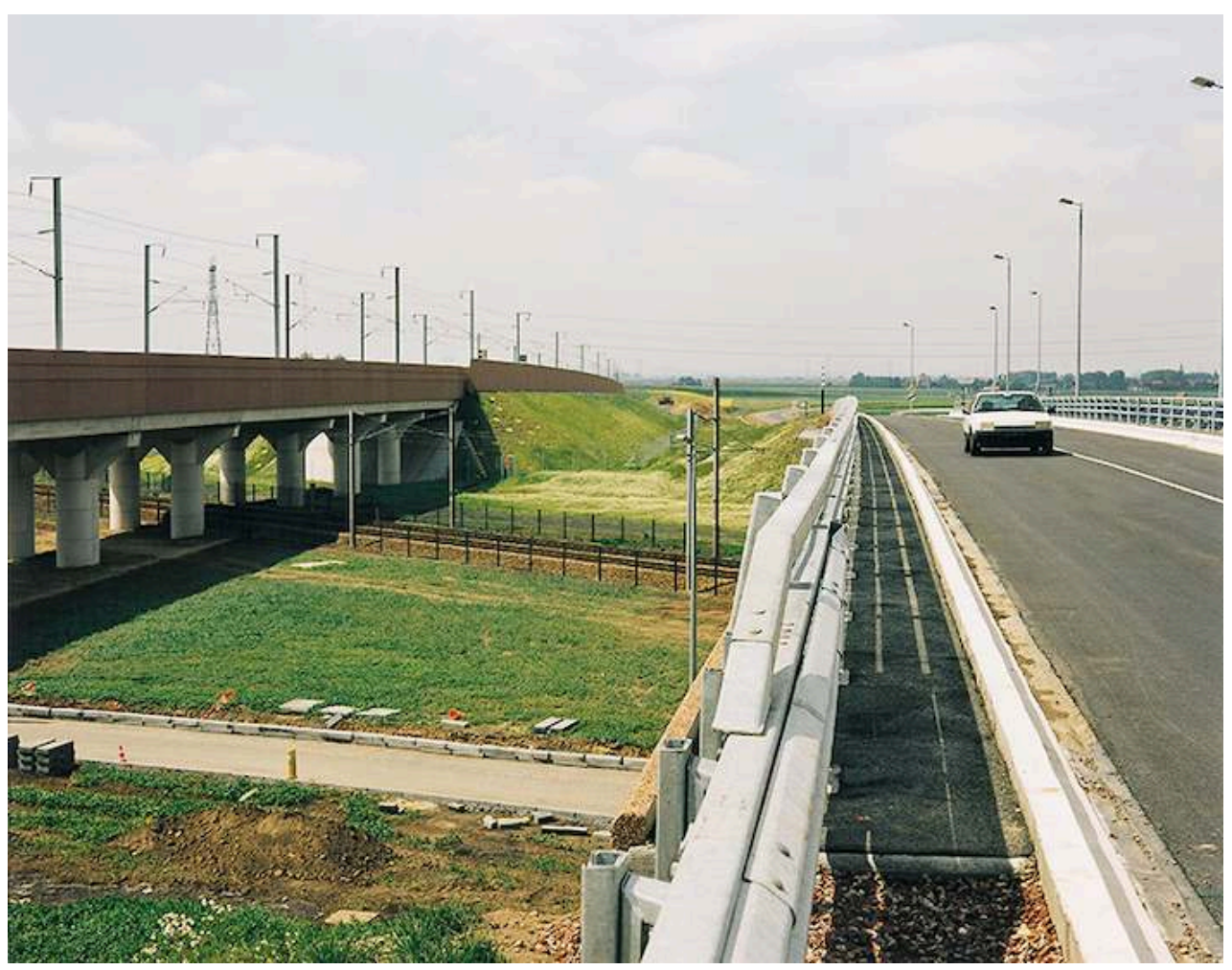

Itinéraire $n^{\circ} 4$, Nord-Pas-de-Calais (Dreal).

Source : Dominique Auerbacher, OPNP. 
Figure 5. Henin-Beaumont - Sortie de magasin d'un centre commercial (04 00501), juin 1993

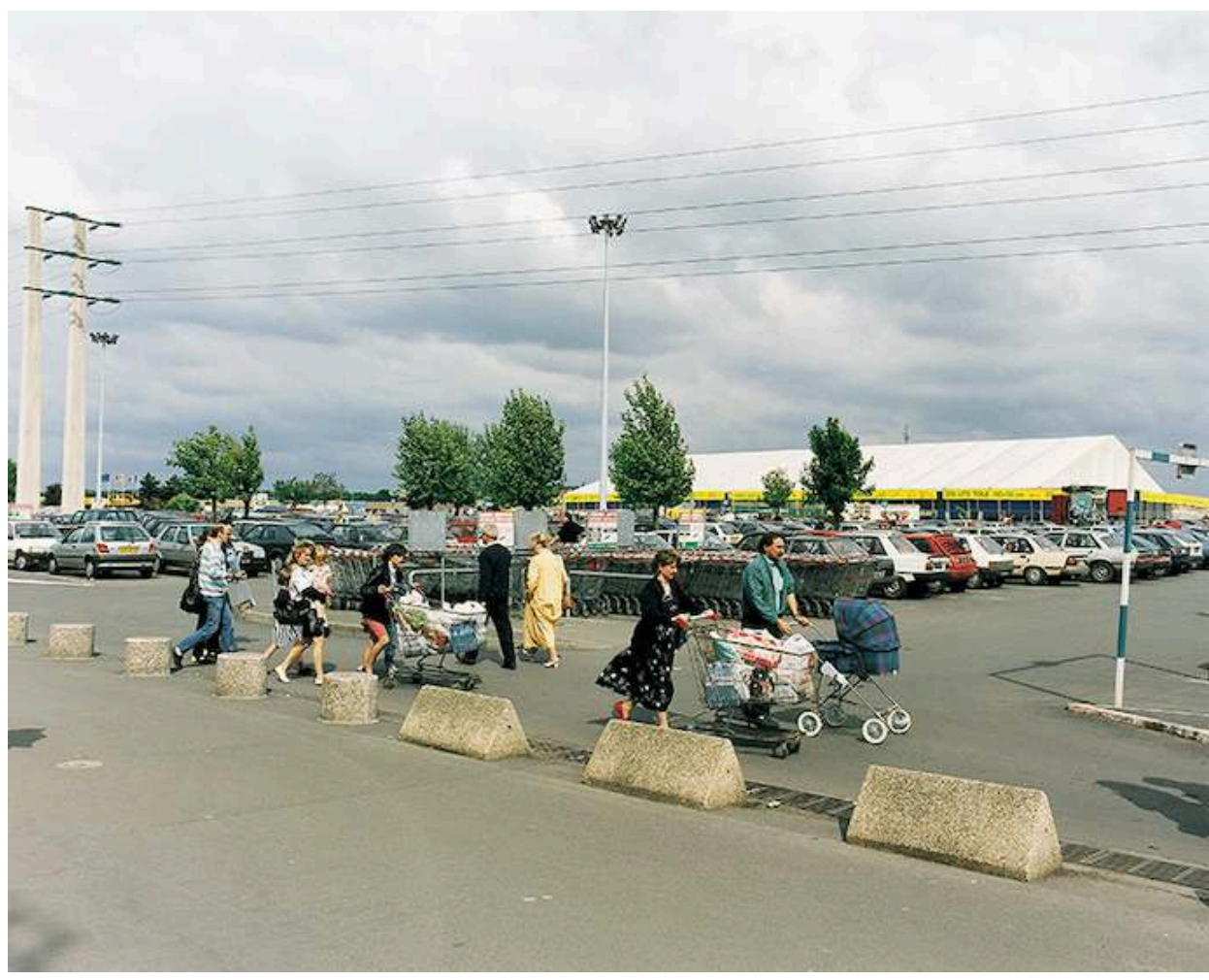

Itinéraire $n^{\circ} 4$, Nord-Pas-de-Calais (Dreal).

Source : Dominique Auerbacher, OPNP. 
Figure 6. Dourges - Lieu-dit Les Douchis (04 012 01), juin 1993

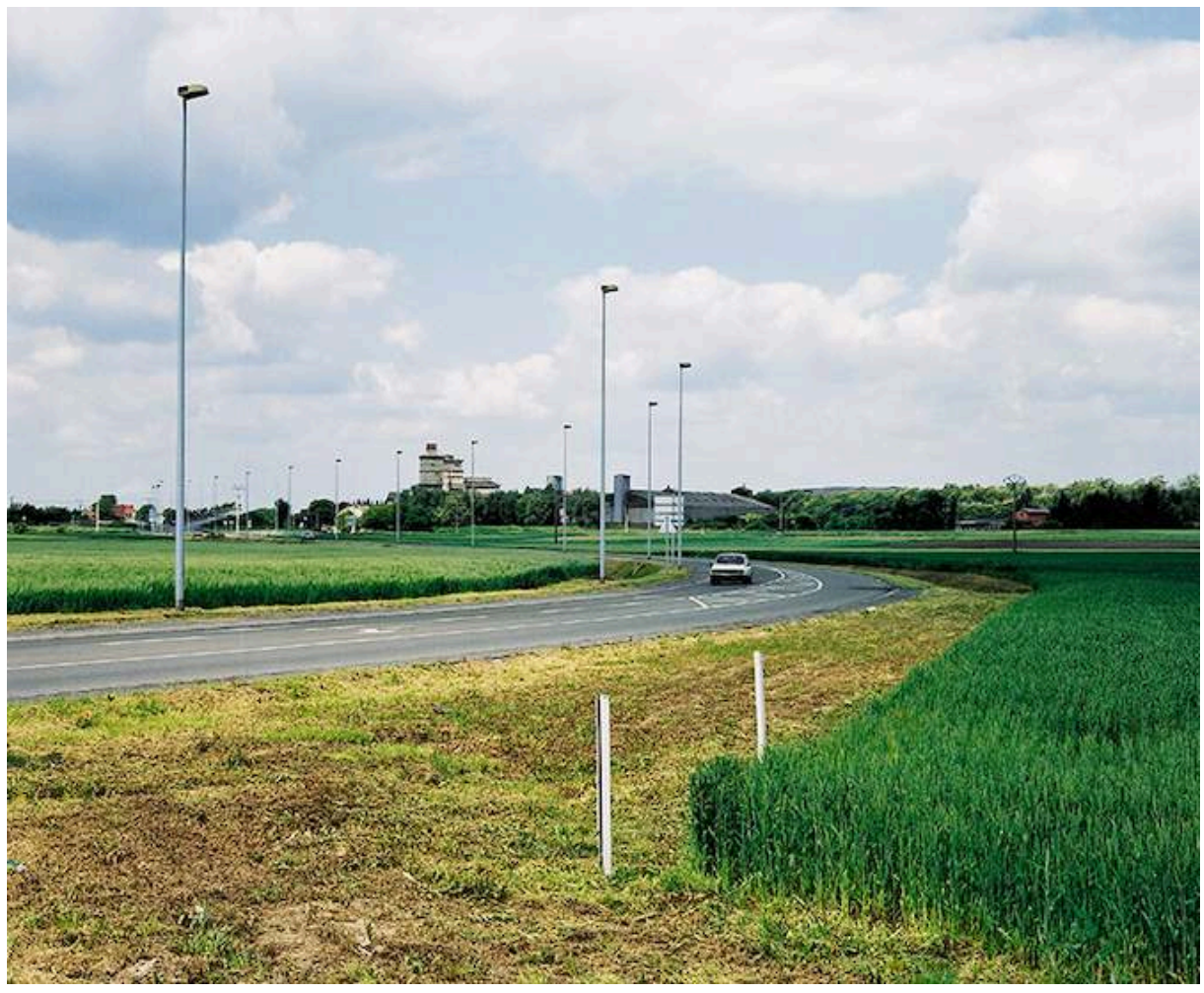

Itinéraire $n^{\circ} 4$, Nord-Pas-de-Calais (Dreal).

Source : Dominique Auerbacher, OPNP.

7 L'accent mis sur les aspects visibles, sensibles et subjectifs du paysage peut surprendre, de la part d'un représentant du ministère de l'Environnement; et plus encore, la présentation simple, voire simpliste, du paysage comme "système de lecture visuelle de l'état de l'environnement ». Est-il suggéré que le paysage aurait des propriétés sémantiques, serait comme un texte à lire? Pour éclairer son positionnement, Jean Cabanel précise, en note, faire référence à la «notion immatérielle du paysage la plus communément admise tant par les géographes Ferrier, Bertrand, Berque, que les sociologues Sansot ou des paysagistes tels que Lassus ", s'affiliant ainsi à un courant de pensée qui se développe alors en France $^{5}$ sans pour autant, peut-être, en saisir précisément les positions philosophiques et esthétiques. Néanmoins, signalons que si ce passage insiste surtout sur la dimension immatérielle et sensible du paysage pour expliquer l'emploi de la photographie et légitimer l'implication de «photographes de haut niveau ", le texte n'oblitère pas la considération de la dimension matérielle, pour laquelle sont proposés des relevés phytosociologiques, un tableau de bord statistique et un centre de documentation sur le paysage. Finalement, cette première annonce du projet, malgré un caractère quelque peu caricatural, propose les premiers points clés : le suivi de l'évolution des paysages dans le temps, l'articulation des données sensibles aux informations relevant de la dimension matérielle du paysage, et enfin l'appréhension du paysage comme "vecteur médiatique ", préfigurant peut-être la perspective la plus fructueuse de l'OPNP.

En octobre 1991, le ministère de l'Environnement annonce le lancement de l'observatoire photographique national du paysage. Cette démarche est, rappelons-le, fortement exploratoire et fabrique ses outils et objectifs en même temps qu'elle 
expérimente. Son principe et sa forme sont résumés par Marie-Odile Guth, alors directrice de la Nature et des Paysages au ministère de l'Aménagement, du Territoire et de l'Aménagement, en ouverture du colloque "Itinéraires Croisés ", tenu en 1999 à Rochefort. Il s'agit de "réunir dans une même démarche un territoire parcouru selon un itinéraire, un photographe de talent et un comité de pilotage local rassemblant les acteurs de l'aménagement. Sur chacun des itinéraires, à l'issue d'une réflexion de 3 ans fondée sur les premières prises de vue, 40 points de vue représentatifs de la question des paysages ont été retenus et font l'objet de rephotographies annuelles dans un cadrage et à une date identique ${ }^{6}$. " La démarche vise à faire un état des lieux en France et en région, à observer pour comprendre l'évolution des paysages, à informer et à sensibiliser : outil d'aide à la décision, outil pédagogique, outil de projet (Meeddat, 2008)... Autant d'objectifs comme des hypothèses projetées, qui concorderont d'ailleurs avec ceux de la Convention européenne du paysage.

\section{Fondements théoriques et pratiques d'une expérience pour le paysage}

9 Justement, de quelles hypothèses ce programme, fait d'une imbrication de méthodes, est-il le fruit ? Quel en est le socle conceptuel, même non formulé, en matière de pensée du paysage, appliquée à la photographie, par la photographie? Quels corrélations et écarts peut-on voir entre le projet et son application, la rhétorique et le terrain? Quel héritage a été reconduit et transformé, depuis les premiers itinéraires jusqu'aux observatoires locaux actuels?

\section{Projet pour le paysage, ou le paysage en projet}

D'abord, ce programme est constitué d'une rhétorique portée par diverses actions de communication : une médiation sur les territoires évidemment, avec l'encadrement des itinéraires par la mission (devenue Bureau des paysages); mais aussi des actions de diffusion, comme en témoignent l'exposition coproduite avec la Cité des sciences et de l'industrie en 1994 et surtout la revue Séquences Paysages lancée par le ministère (qui n'aura que deux numéros, en 1997 et 2000). Il s'agit de faire connaître et de promouvoir, mais aussi de légitimer le projet dans et par différents domaines, le maître d'ouvrage souhaitant le voir interrogé par différents champs de la recherche. Par exemple, le colloque "Itinéraires croisés » tenu en 1999 à Rochefort a-t-il convié des paysagistes, des géographes, un théoricien de l'art, une philosophe, un professeur de sémiologie de l'image, etc.

11 Et cette rhétorique fait déjà projet: alors que les textes législatifs mettent en mots, l'OPNP, s'appuyant sur la dimension performative de la représentation, met en images pour faire exister et normaliser un déplacement vis-à-vis du paradigme paysager moderne et de sa politique. D'une conception "patrimonialisante ", pétrificatrice et normative fortement associée au concept de "paysage national " (Jakob, 2007), on passerait à l'idée d'un paysage démocratique. On ajouterait à nos "paysages patrimoines" nos paysages quotidiens, faisant des "dynamiques paysagères ». Le paysage ne serait plus réservé à une élite mais devient bien commun expérimenté quotidiennement, ce que formalise d'ailleurs l'itinéraire photographique produit en vue piétonne depuis des lieux publics (la route, principalement). 

régulièrement, fait fondamentalement écho au besoin humain d'archiver le réel. Héritier de l'association originelle de la photographie, du territoire et de l'institution par la commande initiée en 1851 par la commission des Monuments historiques avec la Mission héliographique, l'OPNP renoue avec les origines de l'inventaire photographique comme action de "conquête visuelle", physique et symbolique (Rouillé, 2005, p. 124) : il s'agit d'illustrer pour mieux ordonner et agir sur le réel. Mais la portée de ce procédé archivistique va au-delà de l'expérience mémorielle, comme le rappelle Arnaud Claass dans une réflexion portée par la pensée de Jacques Derrida dans Mal d'archives. L'archive n'est pas qu'opération neutre de « rangement » mais également acte de création : il s'agit, en décidant d'un commencement, de définir une origine et de fonder son objet, de faire acte d'autorité, «en tant qu'acte de consignation et d'assignation, l'archive est une manière de dire et lire la loi » (Claass, 2013, p. 31). Ainsi, l'OPNP, en répertoriant l'état des paysages à partir d'une première image, définit un état de référence, institue d'une certaine manière la réalité et la norme.

13 Et s'il s'agit d'abord d'imager les différents types de paysages (agricole, urbain, périurbain, littoral, etc.) et problématiques associées en fonction de visées techniques, il est surtout question de proposer des paysages dans lesquels encourager les gens à se reconnaître, de répondre, par le vecteur artistique, à une demande sociale formulée ou non formulée (Luginbühl, 2001). Cet objectif identitaire est énoncé dans les premiers supports de communication de l'OPNP et rappelé ensuite à plusieurs reprises, notamment par Caroline Mollie-Stefulesco en 2000 en introduction du second numéro de Séquences Paysages : "Confrontés aux réalités du terrain, engagés dans un dialogue avec les aménageurs, ces artistes faisaient la preuve de la qualité de leur intuition, de leur capacité à traquer dans le paysage les signes révélateurs des valeurs d'une société, de ses aspirations, de ses dérives. Leurs images font office de miroir en renvoyant au regard ce qu'il évite souvent de voir ${ }^{7}$." Revendiquant toujours cette fonction identitaire, l'OPNP se projette comme outil pour formuler et exprimer une demande, ainsi que comme support de débat: "L'observatoire est, pour les aménageurs professionnels, administratifs et politiques un outil de projet pour demain. Il est également un support de débat. En incitant le grand public à accepter qu'il y ait changements, il l'invite à choisir les paysages dans lesquels il pourra se reconnaître ${ }^{8}$. " (Mollie-Stefulesco, 2000, p. 4)

Plus encore, entre inventaire et invention, état des lieux et projection, sa portée symbolique est la réactualisation des modèles paysagers et donc du paysage lui-même. En effet, la conception esthétique d'un paysage par la photographie (parmi d'autres médiums) contribue à la constitution d'un phénomène complexe d'enrichissement du corpus mental individuel et collectif, à la production de références permettant à la fois de définir le paysage tel qu'il est, et d'en mettre à jour le modèle visuel, les propriétés prototypiques, donc de se projeter : finalement, à renouveler les modèles paysagers qui permettent d'ordonner et d'apprécier un paysage (Luginbühl, 2001). L'OPNP, programme institutionnel, renouvelle ainsi méthodiquement une manière de voir et de représenter et contribue à répondre, du moins à faire écho, à la crise des modèles conceptuels et esthétiques du paysage. 
Figure 7. Rampe de la mairie vers la place (09 034 01), octobre 1997

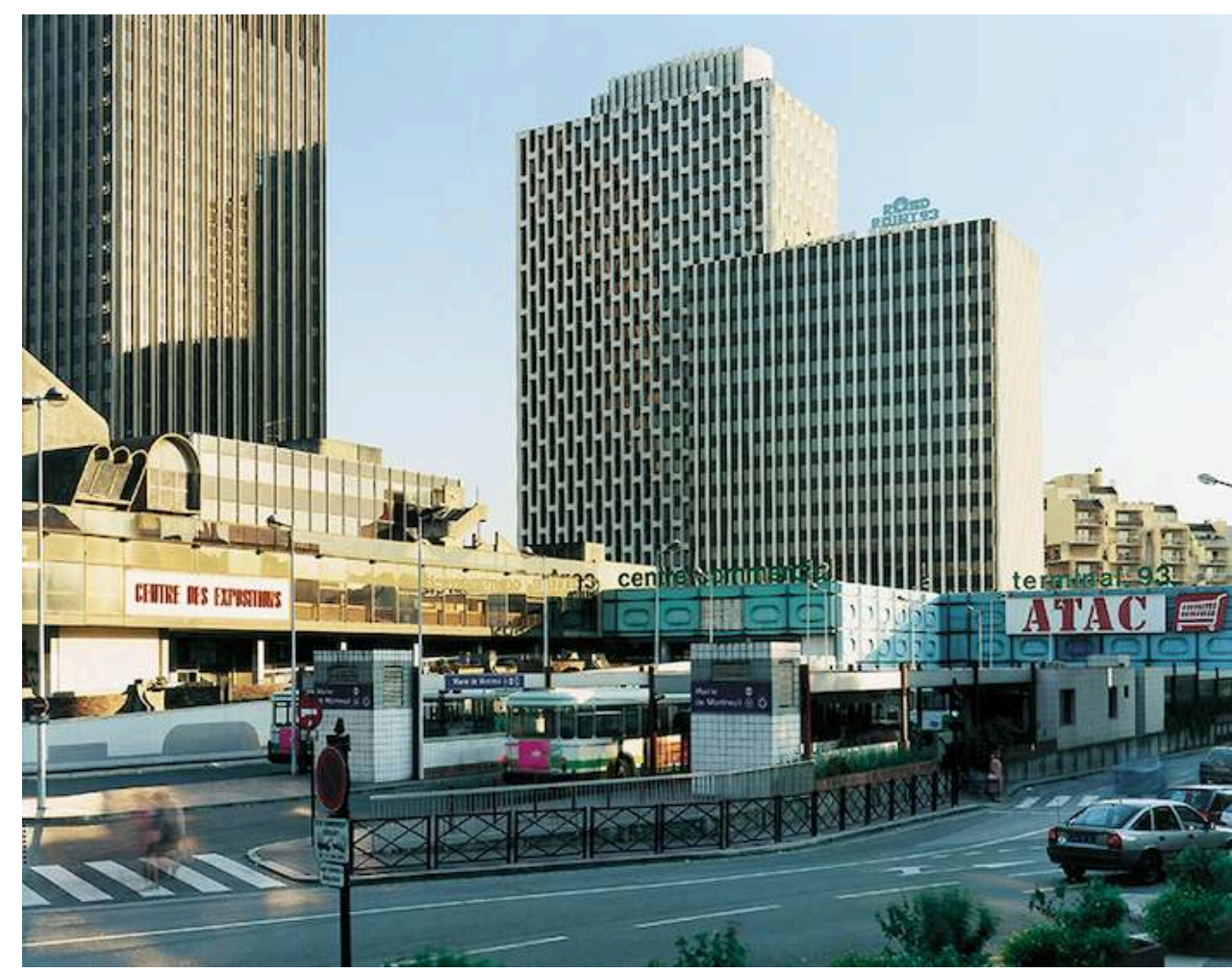

Itinéraire $n^{\circ} 9$, ville de Montreuil.

L'OPNP, tout en souhaitant proposer des documents à visées d'analyse et de médiation, tente d'envisager le renouvellement de l'imaginaire collectif par la prise en compte des nouveaux motifs paysagers. Ainsi, les photographes portent leur regard sur les paysages urbains et périurbains, le « paysage électrique et infrastructurel », ou encore les nouvelles figures de la ruralité.

Source : Anne Favret et Patrick Manez, OPNP. 
Figure 8. Bullion - Les Valentins - D149 à l'entrée du village (01300101), octobre 1992

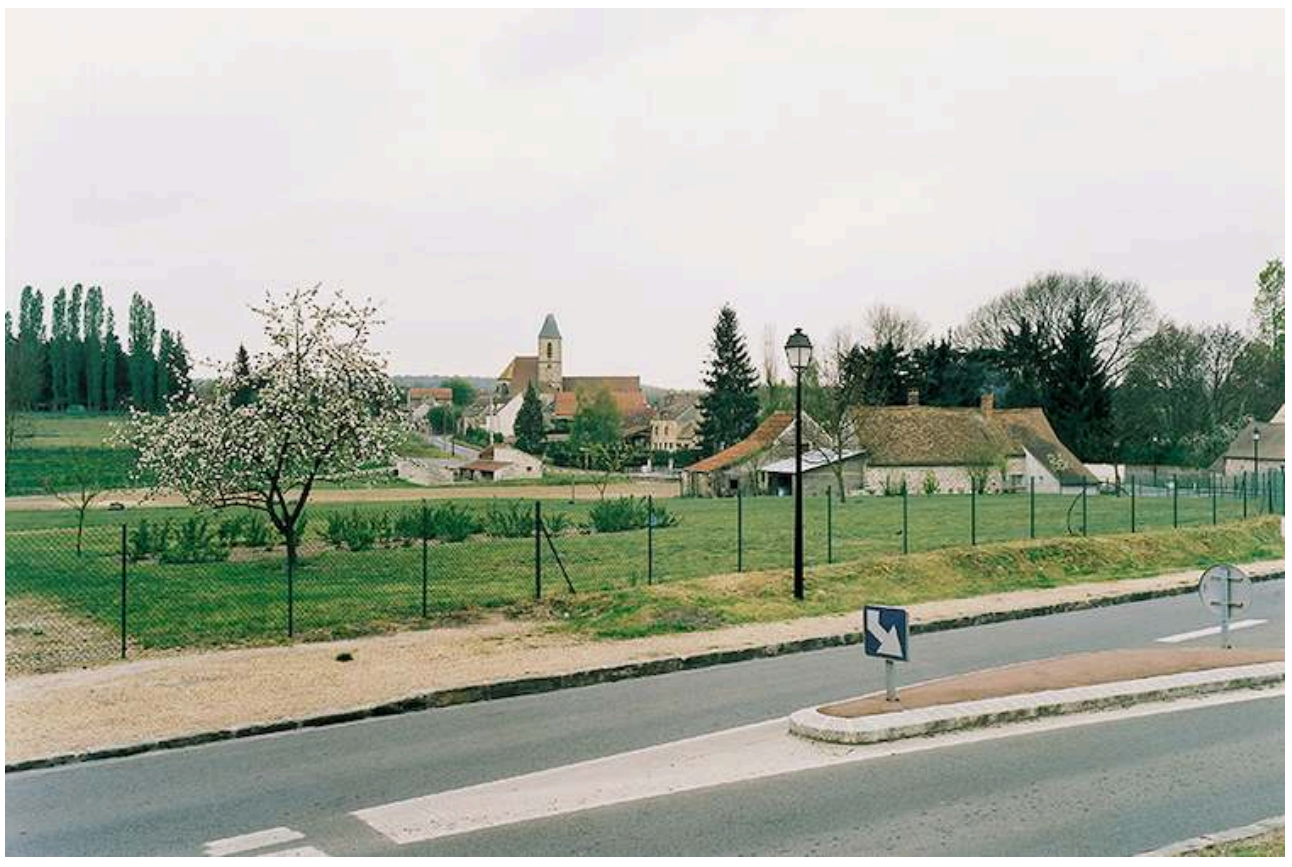

Itinéraire n¹3, Parc naturel régional Haute Vallée de Chevreuse.

Source : Gérard Dalla Santa, OPNP.

Figure 9. Aiglun Mallemoisson (10 030 01), octobre 1996

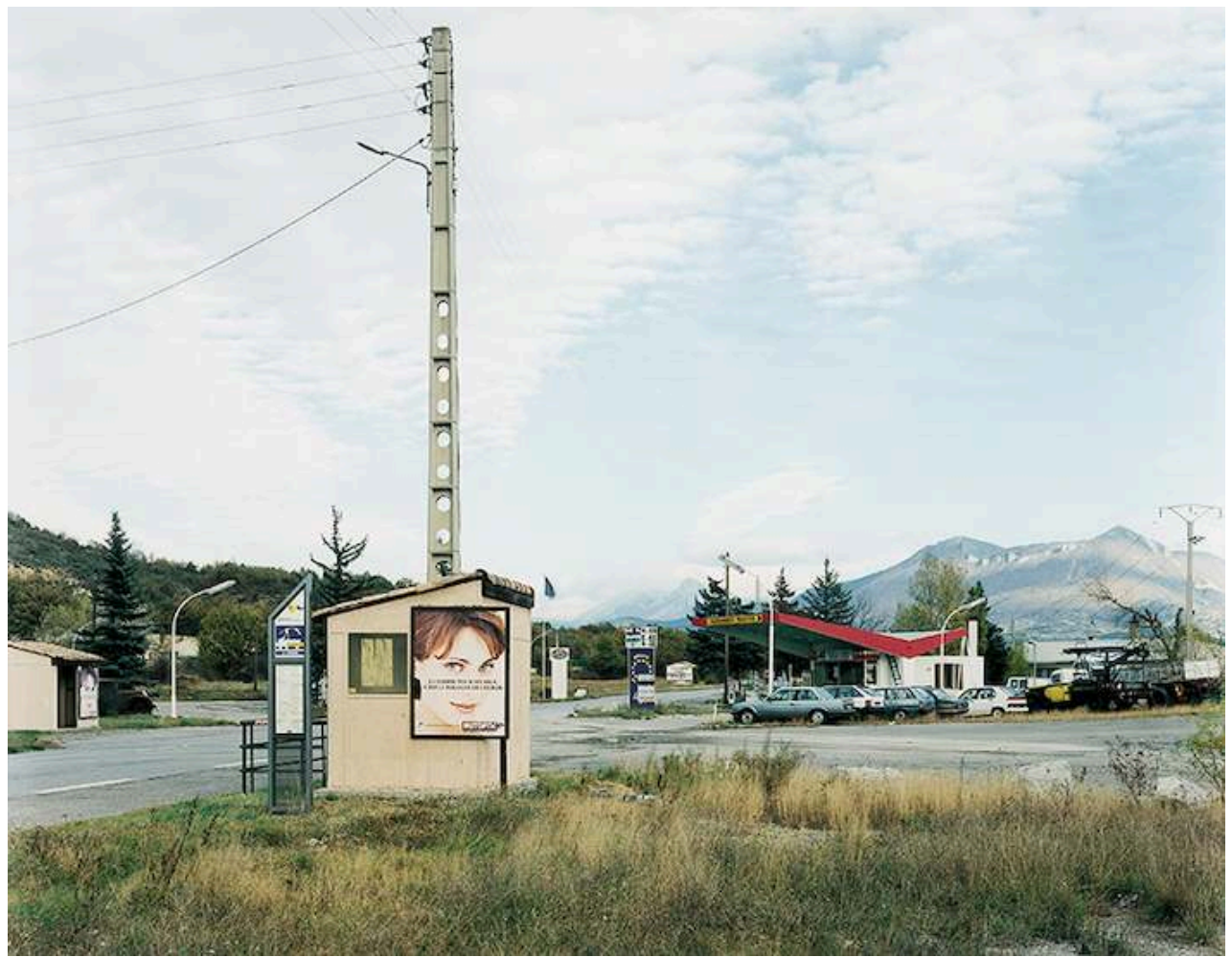

Itinéraire $n^{\circ} 10$, vallée des Duyes et de la Bléone (Dreal Paca).

Source : Gilbert Fasteneakens, OPNP. 


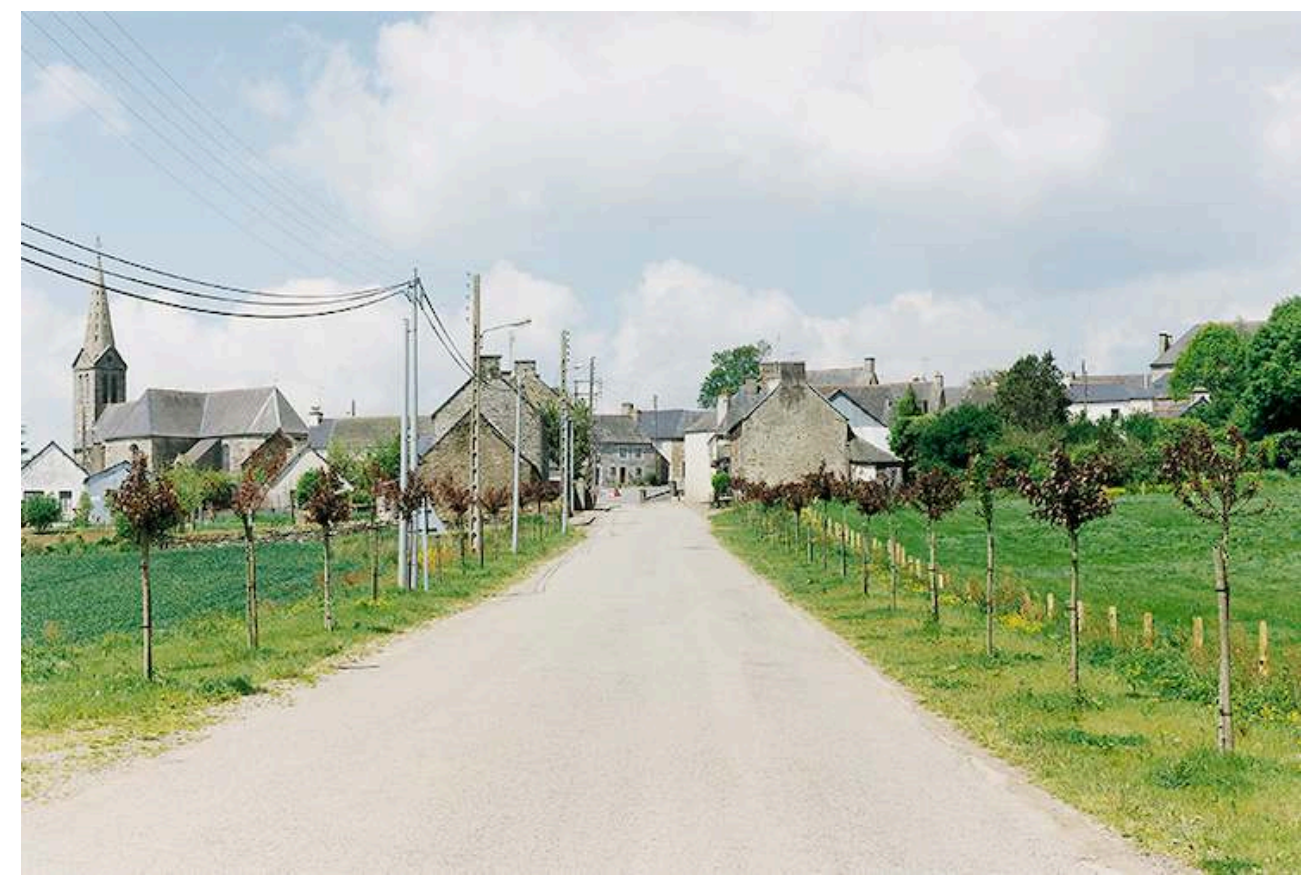

Itinéraire $n^{\circ} 5$, Côtes d'Armor (CAUE)

Source : Thibaut Cuisset, OPNP.

S'appuyant sur ces fonctions socioculturelles, la démarche de l'OPNP tente de penser le paysage comme média. En effet, en plus d'une mise en image (mentale et matérialisée) pour identifier et s'identifier, le paysage, comme expression d'un collectif, matériau et vecteur du sentiment d'appartenance et de l'identité territoriale (Sgard, 2010), semble être un média vers des problématiques matérielles autant qu'un média entre les acteurs. Avec un itinéraire photographique, on produit des images pour débattre, négocier, trouver un consensus et construire un projet de territoire. Le paysage est ainsi envisagé comme "paradigme de médiation entre l'espace et la société" (Donadieu, 2007) ; et une pensée du paysage comme «enjeu et instrument " (Dubois, 2008) est donc esquissée, comme préfiguration de réflexions qui seront développées ultérieurement dans les sphères de l'aménagement. Aussi, l'objet est à l'image de son sujet : reconnaissant la complexité de la fabrication de l'espace et du paysage, il tente de l'articuler dans la production d'un itinéraire, envisagé comme un projet de paysage relevant à la fois du projet photographique, du projet d'analyse des évolutions spatiales, de l'outil pour les pratiques d'aménagement et de l'outil pour le projet politique. La méthode met en avant la forme partenariale: «Un itinéraire photographique doit se concevoir comme un projet né de la rencontre entre un projet de territoire porté par un maître d'ouvrage public et le projet artistique d'un photographe" (Meeddat, 2008, p. 7). L'équilibre dans la collaboration, et même la coproduction, semble être la condition de réalisation d'un projet transversal.

\section{Observation photographique}

16 C'est en effet sur le pari d'une production collective fondée sur les spécificités de l'usage de la photographie comme outil technique et culturel, documentaire et sensible, que repose la démarche: l'enjeu est bien la production d'un Observatoire 
photographique du paysage et Daniel Quesney, photographe chargé du suivi artistique et technique, contribue à la conception de la méthode autant qu'à la formulation d'une rhétorique photographique. La première caractéristique exploitée du médium est son aptitude à produire de la visibilité, une visibilité autre, intensifiée: regarder directement autour de soi n'est pas comme regarder une photographie : une mise à distance est produite, qui permet d'observer les informations selon une autre modalité. Décrivant la force d'évidence de la photographie, Arnaud Class dit qu'elle permet d'« étaler frontalement devant soi des objets comme indices de phénomènes plus vastes qu'eux, et comme indices les uns des autres » et d'«observer les apparences comme lieux pour des analogies et comparaisons » (Claass, 2013, p. 40). Sans faire référence à l'OPNP, en évoquant la force métonymique de la photographie, il formule un des enjeux de la méthode: l'échantillonnage et la représentativité. Au-delà de cette force documentaire, selon la réflexion développée par Daniel Quesney, le processus photographique échapperait par définition à son auteur, "qui n'est maitre que des protocoles » (Quesney, 2008). Et si l'œil et la mémoire humaine sont sélectifs, le procédé photographique ne le serait pas: "autre chose a été "fixé" par le procédé photographique, globalement, au-delà de l'intentionnalité visible et formulable de cet auteur » affirme le photographe dans l'article "L'intuition des photographes » publié dans le premier numéro de Séquences Paysages. Certes, l'appareil capte le visible au-delà de ce qui a été repéré dans l'objectif, mais il convient de prendre en compte les opérations de l'auteur et de relativiser cette conception orientée presque exclusivement sur l'appareil : choix d'un cadre, d'un point de vue, d'une focale, sont autant de critères qui, ajoutés aux problématiques de l'itinéraire photographique, conditionnent la prise de vue et constituent le projet photographique.

Le potentiel du procédé de l'OPNP repose sur la reconduction et la comparaison d'images permettant de lutter contre l'idée d'immuabilité des paysages. En effet, selon la rhétorique de l'OPNP, à l'inverse de la mémoire humaine, la mémoire photographique mise en forme par la reconduction n'est pas nostalgique et serait alors un remède efficace au "poids de la nostalgie, qui renvoie à une image idéalisée ne tenant pas compte en général de l'éclatement de l'organisation traditionnelle du paysage, ce qui produit des vues hybrides qui ne portent pas toujours de nom, ou auxquelles on n'accorde tout simplement pas le titre de paysage » explique Véronique Ristelhueber, toujours dans Séquences Paysages (1997, p. 86). Là encore, il convient de s'interroger sur la prise en compte, dans ce discours mettant en avant les spécificités du médium photographique, de l'auteur et de son individualité : celui-ci ne risque-t-il pas d'exprimer, malgré l'appareil et le dispositif, sa propre nostalgie à travers son regard photographique? Enfin, un argument met en avant la reconstitution comme déploiement des temps du paysage et mise en lumière de ses complexités/diversités temporelles. "Itinérée » et reconduite, la photographie peut être envisagée comme procédant à des coupes d'espace-temps, autant de coups d'arrêt se distinguant de la vidéo et rendant possible l'observation des éléments mouvants qui font les aspects visibles du paysage. Elle invente une autre notion de durée, ou plutôt reconstitue une durée "autre ». Au-delà de la mise en visibilité des changements, la comparaison diachronique désigne les complexités temporelles, à la fois conceptuellement et pratiquement, indique que l'identité paysagère est constituée par l'accumulation d'états, de phénomènes, d'éléments volatiles. 
Figure 11. Rue François Debergue (09 005 01), février 1997

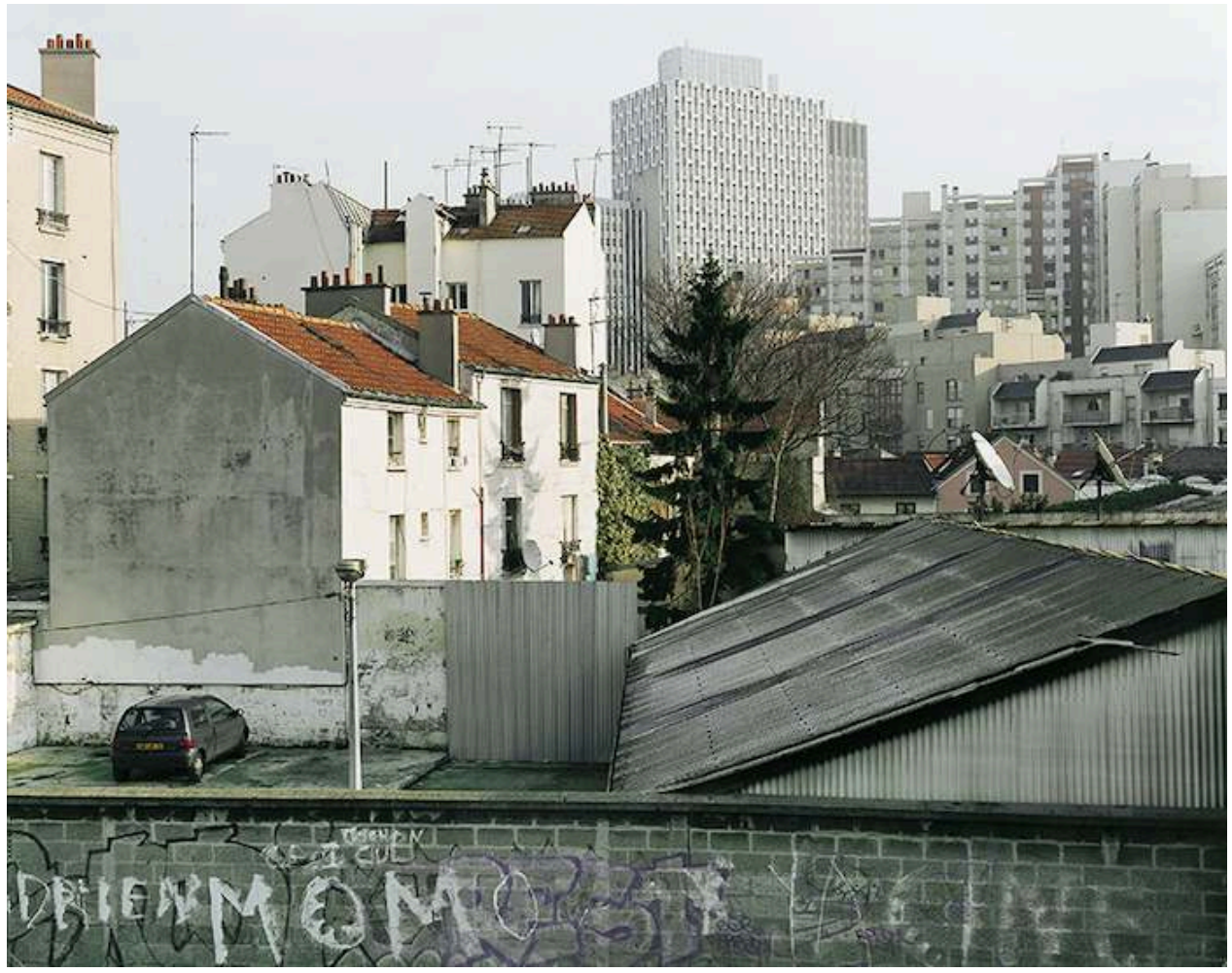

Itinéraire $n^{\circ} 9$, ville de Montreuil.

Source : Anne Favret et Patrick Manez, OPNP. 
Figure 12. Rue François Debergue (09 005 02), février 1998

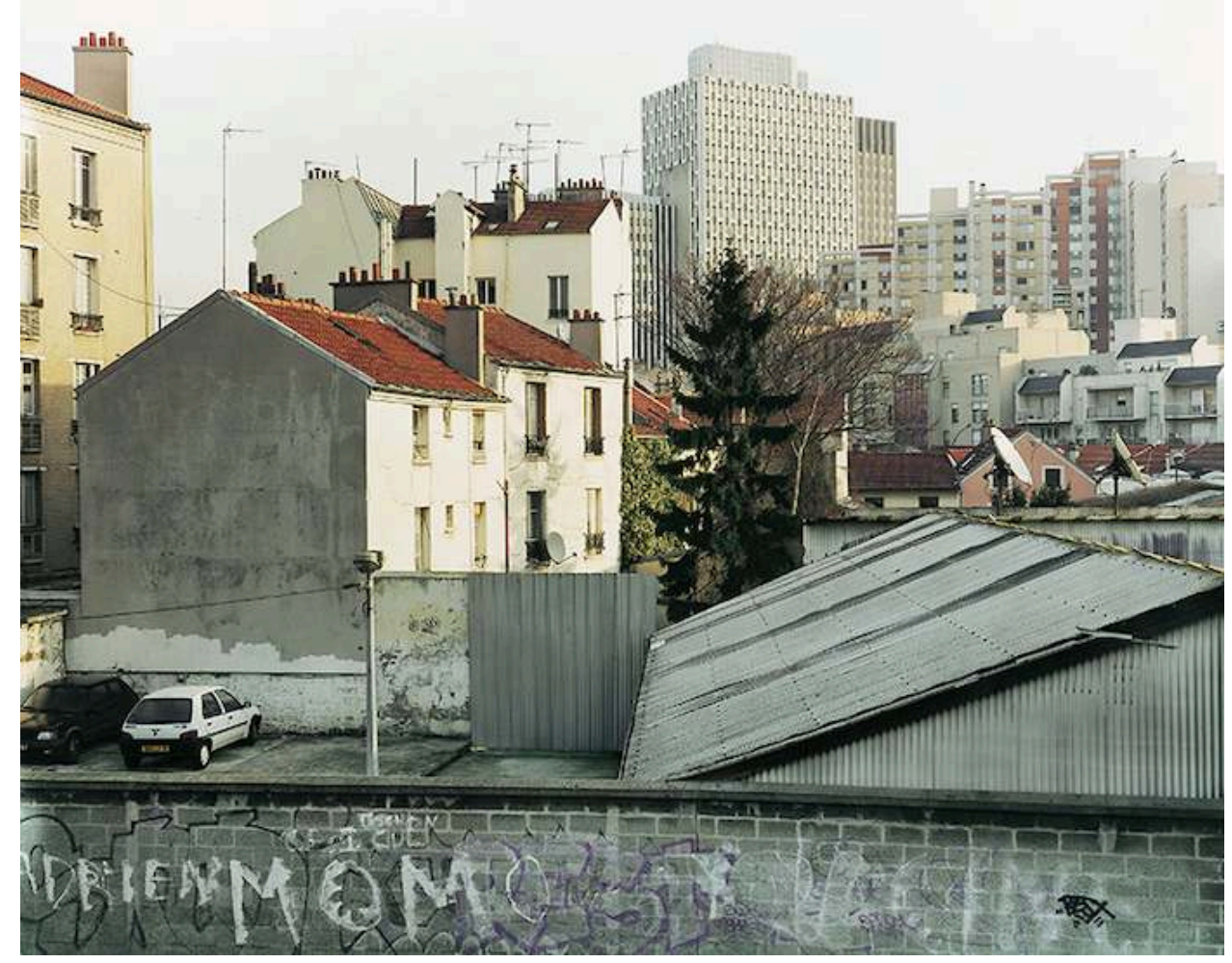

Itinéraire $n^{\circ} 9$, ville de Montreuil.

Source : Anne Favret et Patrick Manez, OPNP. 
Figure 13. Rue François Debergue (09 005 03), novembre 1999

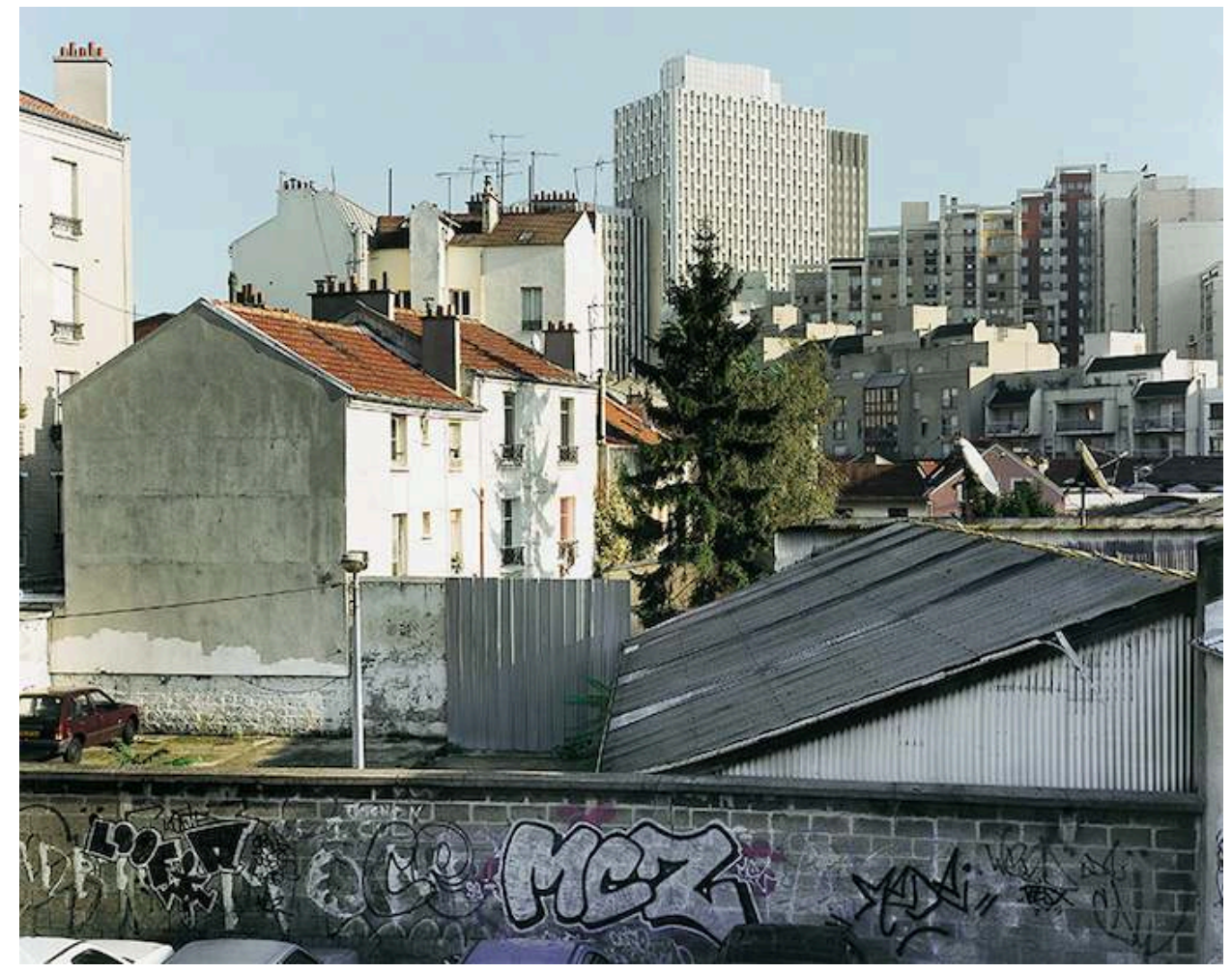

Itinéraire $n^{\circ} 9$, ville de Montreuil.

Source : Anne Favret et Patrick Manez, OPNP. 


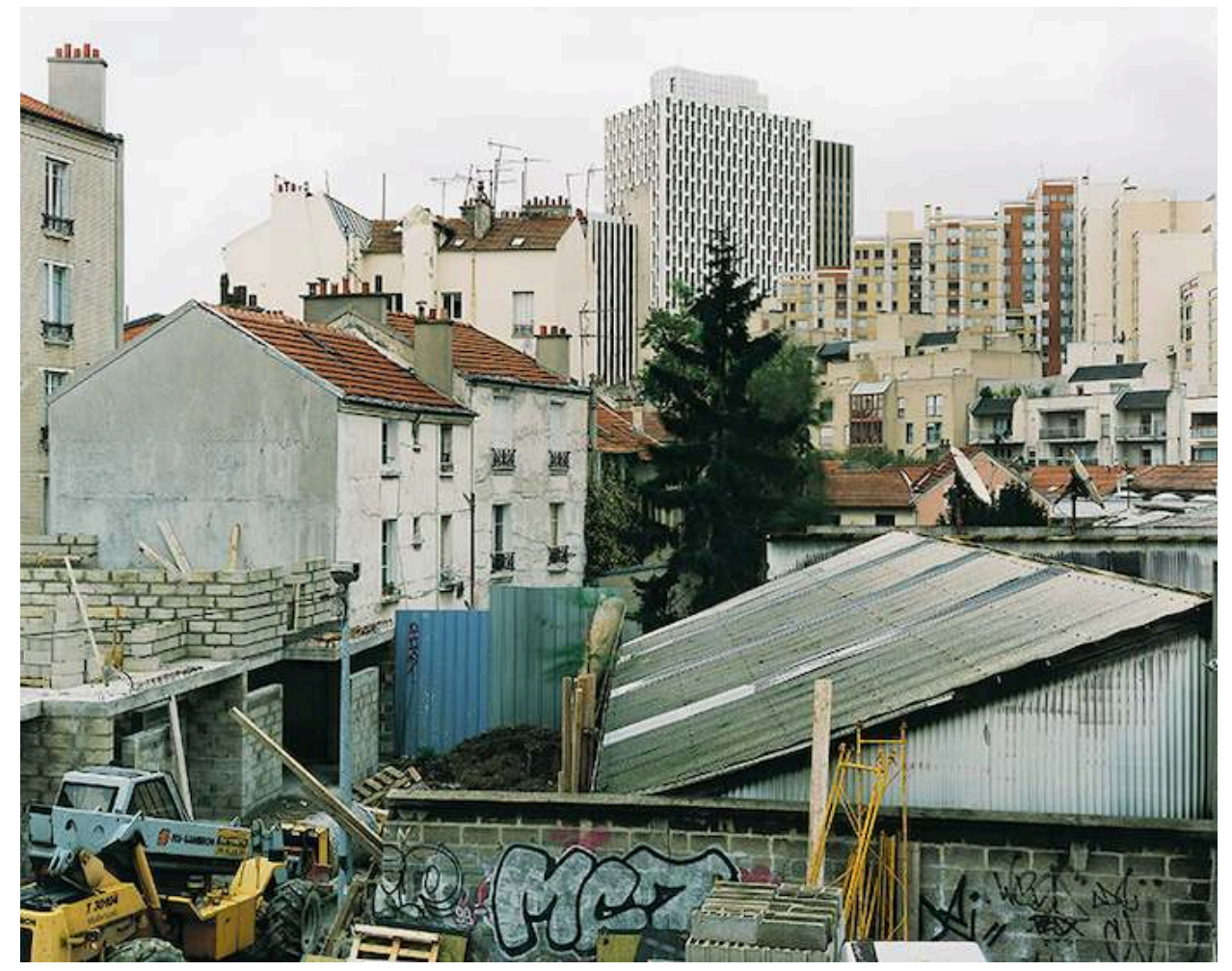

Itinéraire $n^{\circ}$ 9, ville de Montreuil.

Source : Anne Favret et Patrick Manez, OPNP.

Elle évoque alors les différentes échelles de temps, autant que d'espaces, qui font le paysage : temps humain, géologique, écologique, culturel, etc., encourageant à le penser comme une dynamique. Elle nous informe sur le paysage autant que sur notre regard sur le paysage, et nos manières de le représenter.

Dans ce programme, l'usage de la photographie se révèle donc motivé par des spécificités techniques permettant de construire des manières de voir inédites, articulées à des ambitions culturelles fortes, dont l'ensemble est teinté, du moins dans le discours, d'ambiguïtés, voire de paradoxes relatifs à l'appréhension et à la mise en œuvre du dispositif élaboré. Entre volonté d'indexation du réel et reconnaissance du regard photographique, il s'agit de trouver un équilibre sur le terrain.

20 Faisant appel à des photographes reconnus dans le champ artistique pour les premiers et recommandant dans la méthode la collaboration avec des photographes « auteurs ", l'institution exprime un souhait d'affiliation à l'histoire des territoires par la photographie, d'une part, et de reconnaissance du rôle constitutif de la perception et du regard esthétique pour la mise en forme du paysage, d'autre part. «En associant l'approche sensible et l'approche scientifique, je crois que l'OPNP contribuera à l'émergence d'une culture moderne de l'environnement. Les séries d'images du passé apportent une expérience de l'espace, elles ne sont pas destinées à servir de modèle. En revanche, le regard contemporain que les photographes portent sur l'espace nous aide à connaître notre monde » écrit Marc Sanson (directeur de la Nature et des Paysages) dans le premier Séquences Paysages. De nouveau, l'OPNP en tant que rhétorique et programme tente d'articuler la complexité du paysage comme objet culturel, hypothèse construite de réalité, produit d'un regard, représentation in situ et in visu. La méthode 
peut d'ailleurs être considérée comme une combinaison, mise en abyme de dispositifs mettant en exergue le caractère construit de ses objets, le paysage et sa représentation. En effet, le paysage lui-même est un dispositif technico-culturel d'appréhension de l'environnement et la vision du paysage, quant à elle, est permise par la conception d'un dispositif, construction spatio-temporelle, fruit d'une intention esthétique : « une manière de capture du regard » (Bonn, 2008), construisant les conditions du voir. Enfin, la photographie, dispositif de mise en représentation du regard, est elle aussi soumise aux protocoles, donc au dispositif plus général, de l'OPNP qui décuple ainsi les domaines et niveaux possibles d'observation.

L'observation est donc toujours multiple et les formes matérielles et symboliques se combinent. Elle porte sur les éléments qui font le paysage (éléments géographiques, urbanistiques, écologiques, etc.) autant que sur le paysage in situ ou in visu et par conséquent sur la représentation et le regard qui institue en paysage. Il s'agit dès lors de considérer le processus d'«empaysagement" (Briffaud, 2013), qui opère ce processus à partir de modèles, de normes qui, à une époque donnée, définissent le paysage donc le rapport au monde. Un observatoire est toujours un dispositif pluriel portant différents niveaux et champs d'application. Pointant cette complexité (mais sans peut-être être tout à fait conscients des enjeux), les instigateurs de l'OPNP mettent en avant un acteur central du dispositif : le photographe. Si le choix de faire appel à des photographes dits "artistes" relève d'une certaine manière d'un besoin de légitimation culturelle, il est surtout porté par une reconnaissance de leur expertise, comme en témoigne le texte "L'intuition des photographes" publié dans Séquences Paysages, rédigé par Daniel Quesney comme une leçon de culture visuelle (Quesney, 1997, p. 22-26). On s'en remet effectivement à ce savoir regarder et savoir-faire pour articuler l'approche sensible et scientifique, produire des images aptes à répondre à leur fonction documentaire et culturelle, donner à «lire » le paysage dans une proposition visuelle synthétique. Durant le colloque «Itinéraires croisés », en 1999, le sémiologue de l'image Jean Arrouye insiste sur ce point clé des hypothèses originelles du projet : « Les responsables de l'OPNP ont eu raison de faire appel à des photographes confirmés. Il ne suffit pas de regarder pour voir ni de photographier pour qu'un paysage devienne intelligible; pour cela il faut de l'expérience et surtout de l'imagination. » Il ajoute que " la recherche d'une qualité esthétique dans une photo est souvent le moyen de lui conférer une force sémantique » (Arrouye, 1999, p. 92-100). La collaboration prend tout son sens : elle n'est pas la production d'un projet esthétique à ambition artistique d'un côté et d'un projet documentaire à visée technique de l'autre, mais bien la production de l'un, permettant l'autre. Est fait le pari que l'approche culturelle et la production esthétique génèrent l'information et la compréhension du monde.

Et en assignant aux artistes à la fois les tâches de documentation du réel et d'invention, ou plutôt de révélation des paysages, le projet réactualise une tradition de manière originale. Toujours durant le colloque "Itinéraires croisés », Odile Marcel dit que « les photographes de l'OPNP partent à la recherche de ce qui nous est familier pour nous le montrer ». Ils articulent " poétique du savant » et " réalisme du poète », répondent à la fois à la "fonction de synthèse et de projet " (Marcel, 1999, p. 116) et de production d'hypothèses de paysage, et sont reconnus comme acteurs de l'empaysagement des sociétés (Briffaud, 2013). Aussi, par le passage qu'ils permettent entre l'invisible et le visible au moyen de la représentation de ce processus, ils se font médiateurs : entre le paysage et la société et entre les acteurs. Finalement, les choix photographiques, opérés 
par ces emboîtements de dispositifs, affilient l'OPNP à une histoire du paysage ancrée dans l'histoire de l'art, tout en en renouvelant les hypothèses par cette dimension technico-artistique inédite et conceptuelle.

\section{« Paysage-instrument » ou instruments pour le paysage?}

L'OPNP9 est un dispositif à rendre effectif à différents niveaux. Comment combiner l'enjeu du renouvellement des hypothèses de paysage et la production d'un outil de gestion? Comment mettre en œuvre un projet photographique relevant à la fois de l'expérience du paysage par la représentation et de la production d'informations? Par sa rhétorique, le programme formule des enjeux et objectifs qui dépendent de l'articulation du matériel et du symbolique, de la culture du paysage autant que de la gestion spatiale; et il procède pour les atteindre à une "protocolisation " au niveau administratif, technique, photographique, etc. Il apparaît que la méthodologie originelle et plus encore ses transformations mettent en action et en images des contradictions propres à la politique du paysage en France. Aussi, tout en permettant de réaliser des itinéraires, elles "aplatissent» les complexités paysagères et photographiques, et produisent alors des effets «à rebours ». De plus, si la publication de la méthode par le Bureau des paysages en 2008 a permis d'en rappeler les éléments clés (itinéraire photographique, "photographe de talent", reconduction, problématiques paysagères, comité de pilotage), elle a surtout eu pour effet de les fixer et de les canaliser, les faisant passer du statut de " principes » à celui de « règles ». Et ce document, malgré son aspect normatif, ne propose pas de méthode pour rendre opérationnel un observatoire et atteindre des objectifs qui restent d'ailleurs ouverts, voire flous: "comprendre les évolutions des paysages" et "évaluer les politiques publiques ». Les structures porteuses semblent ainsi dans un premier temps séduites par un outil précisément défini et des objectifs prometteurs, mais ensuite démunies en l'absence de méthodologie.

La " méthode ", devenue emblème du programme entier, semble être à la fois condition de possibilités et source d'ambivalences. En effet, elle définit, pour la création et la gestion d'un OPP, les étapes de montage puis la production de documents (carnet de route, grille d'analyse, carte géo-localisée, etc.), garantissant ainsi le bon fonctionnement de l'itinéraire, la reproductibilité des images et la production de données. Elle englobe un cadrage protocolaire qui apparaît comme élément moteur d'une infrastructure iconographique exigeante. Mais elle génère également, surtout par le protocole photographique, des effets non anticipés. D'abord, la forme comme la rhétorique donnent une impression de légitimité scientifique risquant de faire passer pour pertinentes des informations qui ne le sont pas. Et elles encouragent à l'accumulation de données comme autant de pièces à conviction, dans une sorte d'enlisement documentaire : l'archive produit et tourne en boucle (fichiers images et tableaux Excel, indexations, cartes, etc.) oubliant que toute observation doit être portée par un projet. Surtout, cette impression de scientificité, outre un effet d'autolégitimation et d'autonomisation du dispositif, invite à croire à la «fiabilité » de "résultats", ainsi qu'à la possibilité de les mesurer, comme si le regard qui fait le paysage pouvait être ainsi saisi. Au-delà, la caution symbolique apportée par cette «croyance du scientifique » donne autorité autant au procédé d'observation qu'aux 
politiques du paysage qu'il supporte. On constate donc une fétichisation de l'observatoire comme infrastructure administrative, technique, archivistique et photographique qui produit aujourd'hui plus d'archive non photographique que photographique. Cette fétichisation, rigidifiant la «méthode» dans les esprits si ce n'est dans les faits, semble limiter les questions quant à la pertinence et à l'adaptabilité d'une infrastructure qui, pourtant, à son origine, voulait proposer des manières de faire contextualisées et évolutives.

Plusieurs démarches mènent néanmoins des réflexions pour développer un OPP adapté à leurs problématiques paysagères, territoriales, sociales, etc. Mais dans leurs expérimentations pour exploiter cet «outil», elles produisent parfois des méthodes " objectivantes", porteuses d'ambivalences, voire de contradictions. Se confrontentelles, involontairement, aux impossibilités du programme originel? Par exemple, le Parc naturel transfrontalier du Hainaut (PNTH) créé en 2009 l'observatoire photographique transfrontalier des paysages, suite à une réflexion sur la méthode dite «nationale » et à l'élaboration d'objectifs adaptés aux besoins locaux, entre étude technique des évolutions spatiales, représentation d'une identité paysagère transfrontalière ${ }^{10}$ et production d'un outil de médiation plurielle.

Des méthodologies spécifiques sont développées et publiées dans des documents pédagogiques, faisant alors acte de médiation du paysage par l'OPP, pour lequel une photographe-artiste a été mandée, Édith Roux. Particulièrement sensible aux problématiques identitaires et culturelles, envisageant le paysage comme vecteur de valeurs et d'ambitions partagées, le Parc présente dans le guide méthodologique le paysage comme " un grand livre »(PNTH, 2014, p. 2), qui serait à lire à travers les photographies de l'OPP. Or, le paysage est-il un langage, fait d'une grammaire, d'un vocabulaire, d'une syntaxe à déchiffrer? Cette formule semble symptomatique des ambivalences en jeu, à la fois dans la compréhension du paysage comme réalité hybride et la définition d'un OPP comme outil d'analyse. Le document explique dans le même temps que l'OPP «n'a pas pour ambition de devenir un outil scientifique qui décortique en long et en large la sémantique du paysage » (ibid., p. 2). Pourtant, deux méthodes d'analyse ${ }^{11}$ des images sont publiées, l'une grand public et l'autre technique. La première, qui propose de "mettre en mots" les éléments constitutifs des images, envisage le corpus comme vecteur et support de médiation, utilisant les vertus communicationnelles de la représentation photographique : celle-ci, quand elle fait l'objet d'une attention guidée, permet en effet de libérer la parole, d'esquisser des interrogations et réflexions, d'embrayer la narration d'un territoire, de réunir autour d'un projet commun, comme le présente d'ailleurs Vincent Piveteau, lors du colloque de Rochefort, avec l'intervention «La photographie de paysage. Un outil de médiation pour les territoires » (Piveteau, 1999, p. 69-77).

Le volet technique du PNTH développe quant à lui plusieurs méthodes. L'une d'elle consiste à cartographier le périmètre embrassé par le cône de vue d'une photographie afin d'en mesurer les surfaces d'occupation du sol pour reporter ensuite en pourcentages ses différentes natures dans un diagramme. Cet exercice exemplifie tout à fait les formes et conséquences de la «croyance scientifique " produite par le protocole appliqué à la représentation photographique du paysage. Est-il pertinent de mesurer des surfaces sur une photographie de paysage qui est, par définition, perspective et qui, surtout, est le fruit d'un dispositif construit par un regard? Entre surinvestissement de ce qui peut être vu dans une image et aplatissement des 
constituantes culturelles et sensibles de la représentation, cette méthode évoque quelques effets d'une "protocolisation" croissante, à rebours des hypothèses originelles du modèle national. Elle montre par exemple qu'on confond paysage et éléments qui font le paysage : s'il est en effet possible de décrire, d'isoler et d'analyser les éléments matériels constituant le paysage, le paysage lui-même est un ensemble qui ne peut être décortiqué. Parce qu'il ressort de l'esthétique et du symbolique, il échappe aux outils de mesure et de compatibilité ${ }^{12}$. Envisageant le paysage comme produit de multiples regards dans le cadre d'une réflexion sur le paysage en politique, des géographes évoquent cette "aporie du paysage » et formulent des interrogations qui relèvent d'enjeux pour les observatoires : «Comment alors figer la matérialité sensible d'un territoire pour l'intégrer à un processus de développement ? Comment objectiver la diversité des regards pour guider l'appréhension du territoire? " (Sgard, Fortin, Peyrache-Gadeau, 2010).

Plus généralement, ces initiatives développées dans le cadre d'OPP démontrent une faible culture visuelle, et plus spécifiquement de la photographie contemporaine, non seulement du grand public, mais également des professionnels. L'effacement des hypothèses photographiques et des questions de représentation, donc l'affaiblissement du paysage considéré comme phénomène culturel, est à la fois cause et conséquence des contradictions observées dans les pratiques contemporaines : « rappelons que pour lancer l'Observatoire photographique du paysage, priorité a été donnée à la dimension sensible et émotionnelle du paysage » lit-on dans Séquences Paysages (Mollie-Stefulesco, 1997, p. 8). Or, aujourd'hui, la démarche semble d'avantage attendue en tant qu'outil technique qu'en tant que vecteur de culture territoriale par le paysage. Il semble également que la conception d'un OPP comme démarche partagée, réunissant autour d'un territoire une collectivité locale et un photographe pour produire un projet dont la transversalité est notamment assurée par un comité de pilotage, soit de moins en moins effective. En effet, la logique administrative, conditionnée par des impératifs de "résultats", ainsi que l'infrastructure méthodologique portant de plus en plus sur «l'extra-photographique» relèguent bien souvent le photographe du rôle de partenaire à prestataire de services. De plus, faute d'expertise et d'encadrement, les structures porteuses sont amenées à lire l'enjeu de combinaison des objectifs techniques et artistiques non comme un défi mais comme une impossibilité, et à concevoir des attentes contradictoires envers le photographe. En effet, celui-ci est tantôt considéré comme artiste-auteur, dans les actions de communication des maîtres d'ouvrage notamment, où son statut est valorisé ${ }^{13}$, tantôt relégué au statut d'opérateur à qui il est demandé d'illustrer des problématiques selon une forme photographique anticipée, qui a pour effet de normaliser autant l'iconographie que la réflexion.

Après 25 ans de confrontation au terrain en recherche d'équilibre entre fonctions techniques et culturelles, le paysage semble rester insaisissable et sa représentation se dérober à l'instrumentalisation. Lors du colloque "Itinéraires croisés » de 1999, Pierre Donadieu évoque les ambitions techniques de l'OPNP et situe la perspective la plus porteuse : «Si l'on doit observer le paysage, c'est en tant que rapport entre le sens des espaces qui évoluent et celui des représentations qui changent, mais à des vitesses différentes; là est l'essentiel du problème social et politique du paysage dans nos cultures occidentales.» " (Donadieu, 1999, p.32.) C'est justement vers cette appréhension relationnelle du paysage ainsi que vers des usages de la photographie comme dispositif pluriel de réflexions et de médiations, que des initiatives 
contemporaines se tournent. Il apparaît pertinent, pour conclure et ouvrir la réflexion, d'interroger un exemple récent et original.

\section{L'OPP depuis le GR2013, marche vers un récit métropolitain}

Après avoir mené l'OPP du PNR des Monts d'Ardèche et celui de la communauté de communes de la Vallée de l'Hérault, Les Panoramistes (Bertrand Stofleth et Geoffroy Mathieu) créent en 2012 «Paysages usagés, Observatoire photographique du paysage depuis le GR2103 ». Motivés par un fort intérêt pour l'OPNP et ses principes originels, ils en interrogent les possibilités en révisant certains points de la méthode et tentent d'en réactualiser les perspectives par la conception de dispositifs de médiation. Leur production illustre un renouvellement de l'approche du paysage et plus particulièrement une réflexion qui l'envisage comme objet social et politique, à travers une approche esthétique ancrée dans le territoire. Ainsi, nous verrons qu'elle tente empiriquement d'appliquer les modalités du paysage comme «bien commun» développées notamment par Anne Sgard, se confrontant parfois à ses propres limites et aux risques de l'instrumentalisation. Aussi, ce projet photographique mené dans le contexte d'une réflexion sur la métropole permet de penser les statuts du paysage et de son image comme enjeu, ressource, outil, selon une acception aujourd'hui élargie et hybridée du projet de territoire.

31 En 2010, Baptiste Lanaspèze (éditeur et écrivain), mandaté par l'association Marseille Provence $2013^{14}$ pour concevoir un projet culturel, propose un circuit en forme de huit autour de l'étang de Berre et du massif de l'Étoile. Conçu pour être représentatif du territoire métropolitain, il devient le premier chemin de grande randonnée urbain et périurbain de la Fédération française de la randonnée pédestre. Les Panoramistes imaginent un OPP sur ce GR qui, en tant qu'élément d'une infrastructure plus large, leur fournit à la fois un support pratique (partenaires, financements, système communicationnel, etc.) et un sujet aux problématiques fortes: une "métropole d'usages " (Moiroux, 2013, p. 24-26), existant dans les faits mais peinant à intégrer l'imaginaire collectif. Le GR et l'OPP sont l'un et l'autre des démarches qui s'approprient et réactualisent des dispositifs, pour explorer des territoires et des méthodes. En effet, Baptiste Lanaspèze déplace les attendus de la commande d'un objet ponctuel et récréatif à un projet de territoire, vecteur durable de «culture métropolitaine ${ }^{15}$ » et les photographes interrogent, quant à eux, la démarche des Observatoires photographiques.

\section{Réappropriation}

Les Panoramistes transforment la méthode pour tenter d'en ressaisir des enjeux selon eux fondamentaux. En premier lieu, ils bousculent le rapport entre commanditaire et " commandité »; ils ne se veulent pas maîtres d'œuvre ou maîtres d'ouvrage mais plutôt coordinateurs, afin d'encourager le caractère collectif de la démarche. Ils constituent ensuite un comité de pilotage à la manière d'un groupe de réflexion, selon deux critères : réunir des regards et expériences de territoire et penser ensemble les formes, enjeux et utilisations des images. Des artistes du Cercle des marcheurs ayant participé au tracé des 365 kilomètres du chemin sont invités, ainsi que le géographe 
Jean-Noël Consales et la chef de projet photographie MP2013, Floriane Doury. "Le comité de pilotage ne doit pas être le lieu où le photographe se voit ordonner sa mission et évalué, surveillé, contrôlé, validé, mais plutôt lieu d'information, de débat, de transmission, donc déjà de médiation ${ }^{16}{ }^{\star}$ estime Geoffroy Mathieu, formulant ainsi une critique du fonctionnement habituel, où le photographe semble parfois moins envisagé comme partenaire que comme prestataire de services. Pensé comme faisant pleinement partie du dispositif, le comité de pilotage souhaite être ici vecteur de coproduction, notamment d'un cahier des charges qui place au centre la dimension culturelle pour une métropole en mal de représentations.

Ce "retournement artistique " de la démarche d'observatoire par des artistes se revendiquant détenteurs d'un savoir empirique et sensible sur l'espace (Les Panoramistes, Le Cercle des marcheurs) est symptomatique d'un "tournant spatial» (Volvey, 2014) à l'œuvre dans le champ de la création, qui reconsidère la dichotomie art/science et encourage l'approche esthétique plurisensorielle comme mode de connaissance. Mais cette prise d'autonomie interroge du point de vue de la logique et de la durabilité de «L'OPP depuis le GR2013 ». Par exemple, il semble que la pertinence, notamment scientifique, d'un groupe de réflexion composé par les artistes et au sein de leur réseau, et donc privé de pensée et de regard complètement exogènes, soit potentiellement limitée. Il apparaît également que l'accompagnement durable et régulier de l'OPP par son groupe de réflexion, au-delà de la genèse, n'a pas été effectif. Suite à la première réunion du 29 février 2012 précédant les premières prises de vue, le groupe ne s'est réuni que deux fois : le 16 octobre 2012 pour débattre et discuter des premières images et le 15 décembre pour opérer une sélection finale pour un projet supposé se poursuivre jusqu'en 2022. Ces éléments, parmi d'autres, montrent que les deux instigateurs, avec cette initiative artistique dont le principe est la mobilisation citoyenne, se confrontent à un biais du projet, contenu dans sa nature: l'absence d'encadrement institutionnel, notamment par une structure chargée de l'aménagement, qui pourrait le faire résonner, l'exploiter et le pérenniser. Et si « L'OPP depuis le GR2013 » est une production CNAP/MP2013, ces organismes culturels n'en assurent pas en effet les conditions d'exploitation (volet participatif et autres activités de médiation, éventuels usages analytiques, etc.) au-delà des événements de l'année 2013.

L'objectif de l'OPP se veut général: "documenter et faire découvrir la richesse d'espaces injustement disqualifiés, relever les frottements entre ville et nature, renouveler les représentations culturelles de ces espaces, produire les archives d'un territoire "en devenir" et documenter l'inscription d'un chemin dans un paysage » (Les Panoramistes, 2012 ; en fonction des thématiques produites collectivement en réunion et qui apparaissent d'ailleurs comme une réponse, tant dans leur conception que leur contenu, aux problématiques paysagères imposées usuellement. 
Figure 15. Étang de l'Olivier, chemin du Safre, Istres, novembre 2012

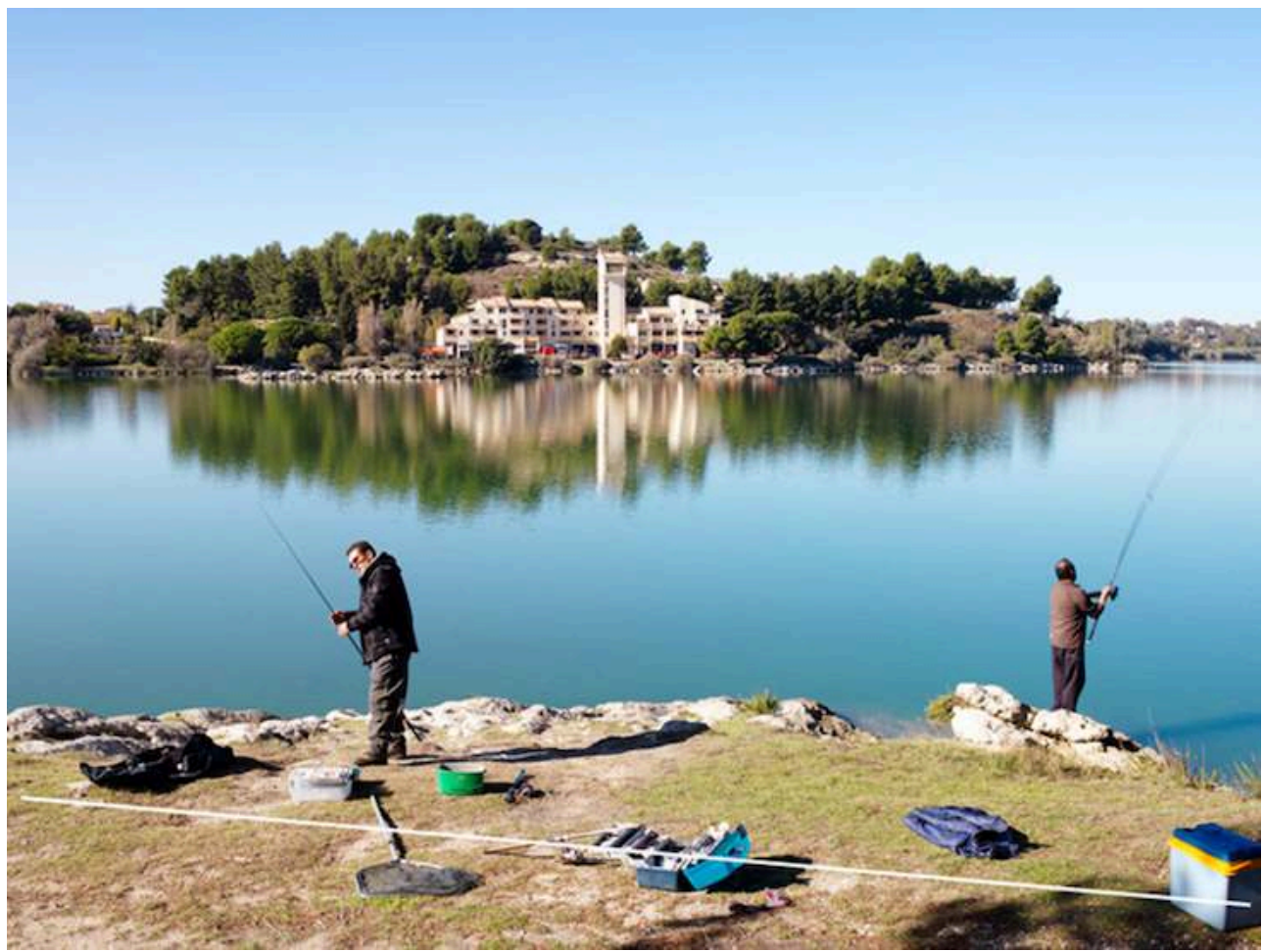

Entre documentation sans concession d'usages et d'appropriations du territoire (loisirs, habitations, industries, etc.) et illustration du patrimoine « naturel » des Bouches-du-Rhône, les photographes tentent de produire un corpus qu'ils jugent à la fois représentatif et porteur de leur esthétique personnelle.

Sources : OPP depuis le GR2013. Bertrand Stofleth et Geoffroy Mathieu. 
Figure 16. Vieux chemin de Lambesc, Salon, novembre 2012

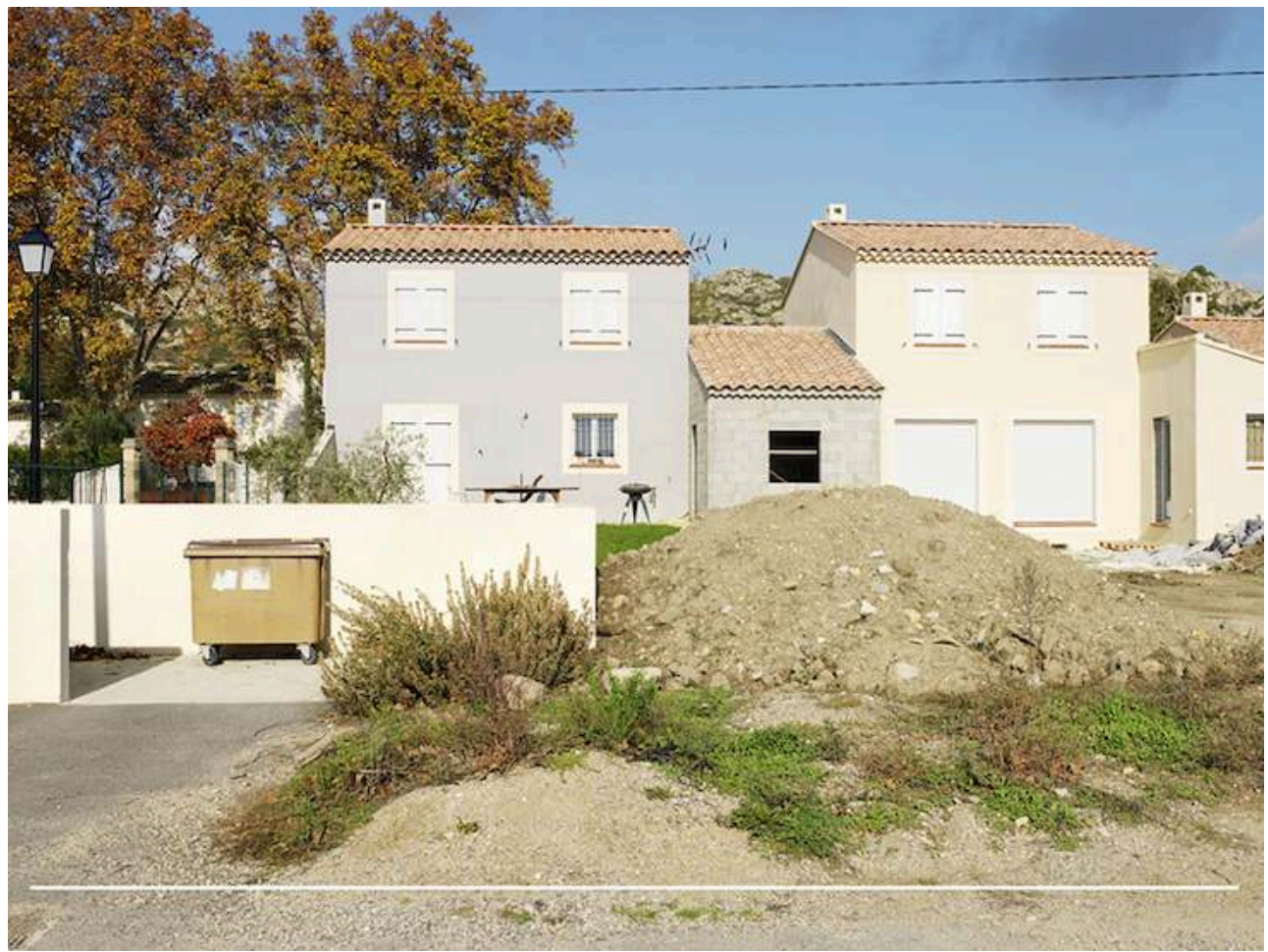

Sources : OPP depuis le GR2013. Bertrand Stofleth et Geoffroy Mathieu.

Figure 17. Étang de Bolmon, Châteauneuf-les-Martigues, mars 2012

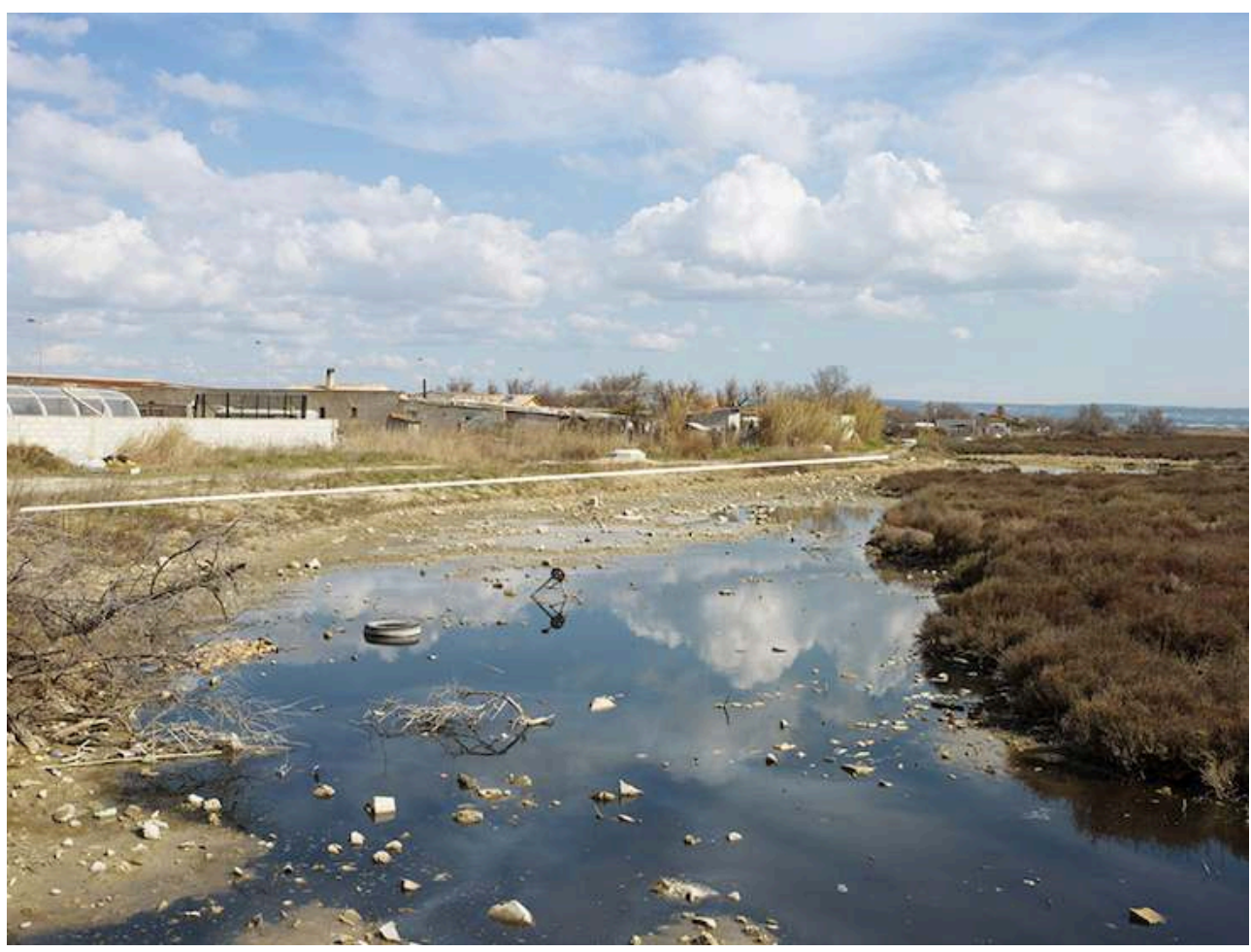

Sources : OPP depuis le GR2013. Bertrand Stofleth et Geoffroy Mathieu. 
Figure 18. Vallon de la femme morte, massif de l'Étoile, Marseille, juin 2012

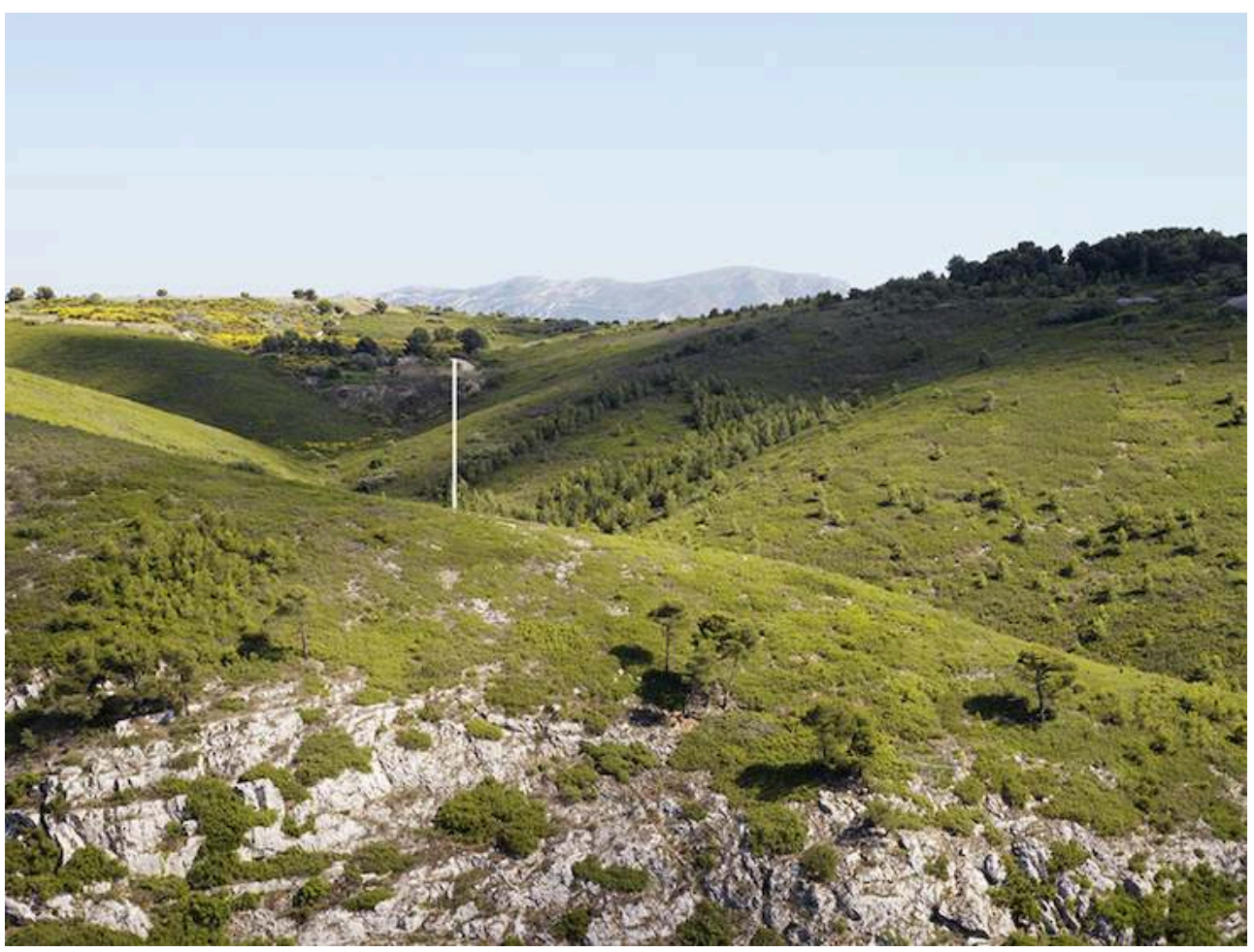

Sources : OPP depuis le GR2013. Bertrand Stofleth et Geoffroy Mathieu.

Structurées en deux rubriques, violence et résistance ${ }^{17}$, elles évoquent la dimension expérientielle et sensible du paysage et fonctionnent comme des embrayeurs de découvertes sur le terrain, plutôt que comme éléments à illustrer, vérifier : "Ces deux thématiques nous ont vraiment nourris. Elles sont assez abstraites et évoquent comment les gens ressentent ces paysages avant de les analyser. Ces dimensions sensibles sont absentes des problématiques données dans le cadre des OPP. Or, justement, c'est la perception qui est le premier vecteur du paysage et de sa représentation : un paysage, ça se traverse, ça se ressent, ça s'habite ${ }^{18}$ » expliquent les photographes. L'action ici menée se distingue à la fois conceptuellement et pratiquement du modèle OPNP et plus particulièrement de la méthode telle qu'elle est mise en œuvre actuellement. En effet, privilégiant l'expérience et la retranscription textuelle et iconographique plutôt que la production d'un corpus problématisé en fonction d'une réflexion préalable, les auteurs pensent différemment le statut de l'objet: de celui d'«outil» au service d'une analyse et d'une politique de paysage, il passe à celui plus général de dispositif de mise en image d'une expérience de paysage et de support de réflexion.

36 La démarche se veut anticonformiste, autant vis-à-vis de la notion de paysage et des paysages métropolitains que de la méthode, perçue comme un « modèle », l'OPNP étant avec la Mission photographique de la Datar une des références de la commande photographique de paysage et un des socles de la culture et de la pratique photographiques des deux photographes. Ils réfléchissent aux points fondamentaux de l'OPNP dans son approche culturelle et privilégient les perspectives de médiation. Il s'agit moins de représenter pour instrumentaliser, vérifier, voire mettre en conformité en fonction d'un modèle, que de découvrir et de réactualiser une image du paysage. Et 
«Paysages usagés » est d'ailleurs revendiqué comme un manifeste en action, résultat d'une volonté d'implication de l'art dans la réflexion et l'action territoriales, illustrant les actuels chevauchements de terrains entre art, sciences et techniques autour des questions spatiales (Volvey, 2014): «Nous voulons être au cœur des enjeux du territoire, être en dialogue avec tous ceux (urbanistes, architectes, écologues, aménageurs) qui les analysent. Nous croyons au potentiel synthétique et de médiation de la photographie. Nous voulons être celui qui fait le pas de côté, qui regarde autrement, qui analyse à rebours, pour proposer des objets plastiques susceptibles de renouveler ou d'enrichir notre vision du monde. Nous voulons sortir du milieu de l'art ${ }^{19}$, retrouver le chemin de l'éducation populaire, jouer avec les codes et les lieux habituels de l'art contemporain. » (Les Panoramistes, 2012).

\section{Médiation, expérience et esthétique}

37 Cette réflexion sur le statut et l'implication de l'artiste, de la pratique artistique liée aux territoires ainsi que de la société civile, s'articule autour d'expérimentations de médiation de et par la photographie. Ainsi, en amont du projet, des marches permettent de présenter l'OPP, mettant alors en œuvre le postulat de l'expérience marchée, à l'origine de l'initiative du GR2013. La photographie devenant un vecteur de découverte in situ du paysage, il s'agit de faire connaître et de sensibiliser, mais également d'impliquer les habitants et usagers. En effet, des cent images de la série, soixante-dix ${ }^{20}$ sont attribuées à des personnes souhaitant «adopter un paysage». Un tirage leur est confié, présentant au recto l'image et au verso sa fiche technique, et elles s'engagent à reconduire l'image pendant dix ans ${ }^{21}$ en vue de sa mise en ligne. Ces adoptants sont formés à la reconduction lors d'événements menés sur le GR par MP2013 ${ }^{22}$ et occasionnellement encore aujourd'hui réunis par les photographes. Si les soixante-dix points de vue proposés n'ont pas été adoptés, un groupe se fédère, dont il s'agirait de trouver les modalités de fonctionnement comme nouveau groupe de réflexion. 
Figure 19. Boues rouges, stadium, Vitrolles, décembre 2012

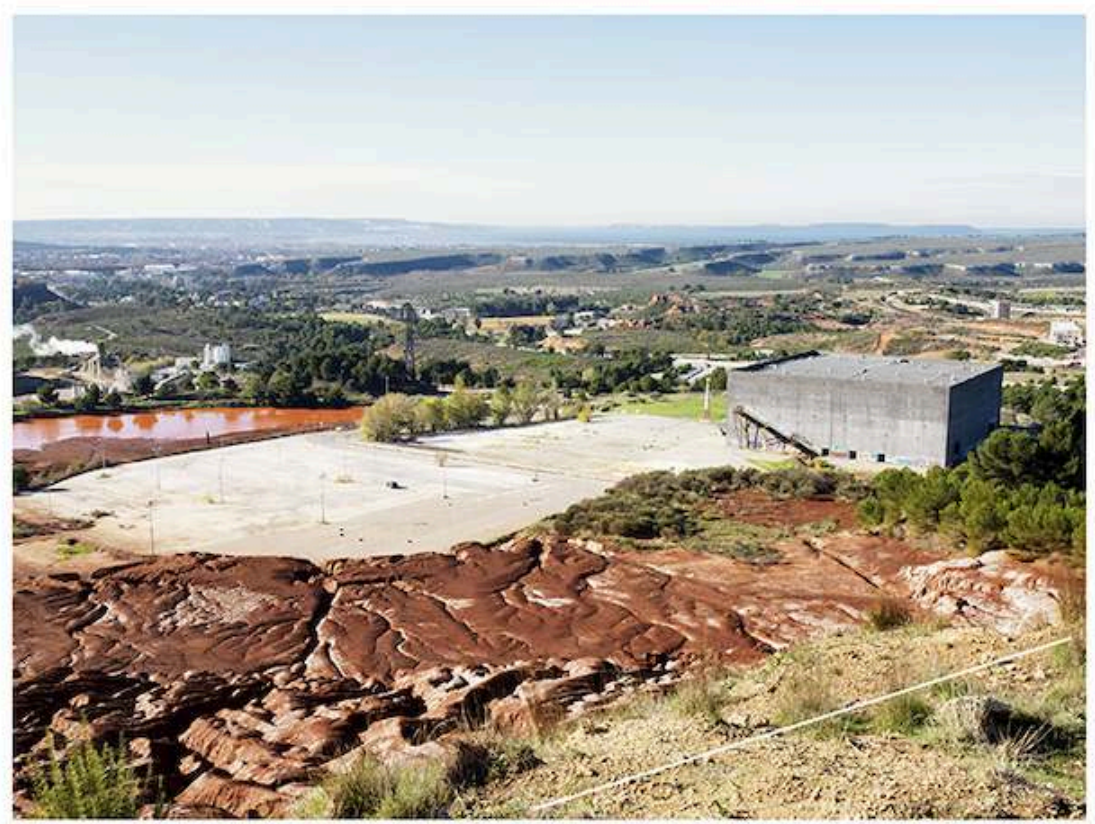

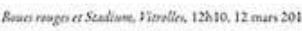

Tirage sous Diasec recto

Sources : OPP depuis le GR2013. Bertrand Stofleth et Geoffroy Mathieu 
Figure 20. Boues rouges, stadium, Vitrolles, décembre 2012
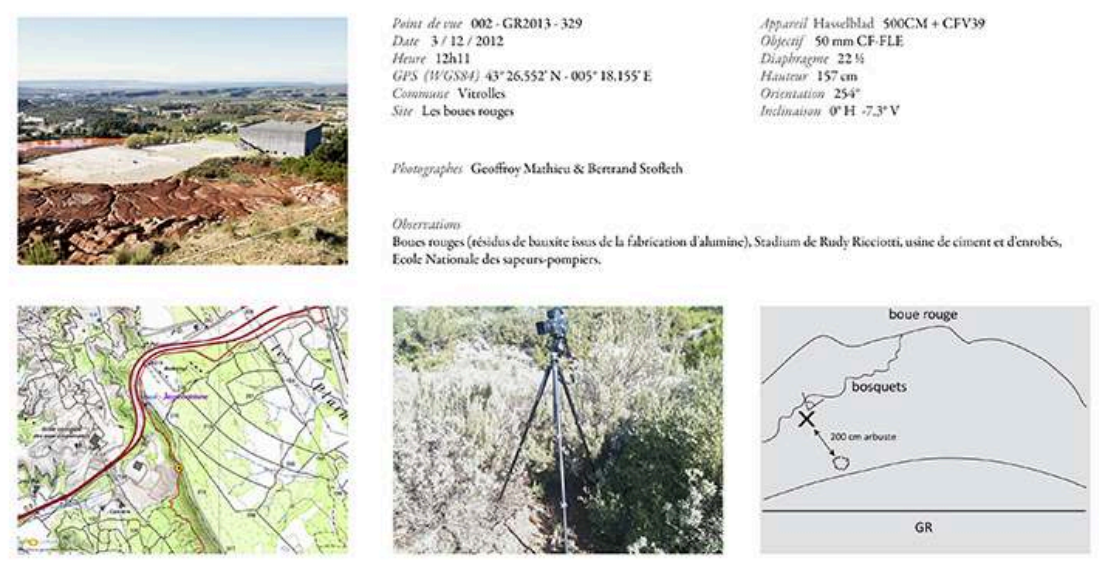

Tirage sous Diasec verso

Sources : OPP depuis le GR2013. Bertrand Stofleth et Geoffroy Mathieu reconduction, une logique de diffusion des images contribue à intégrer le paysage au débat public tout en constituant des productions artistiques : édition d'un coffret de cartes postales, expositions, médiatisations et surtout création d'un site internet. Celuici est à la fois support de diffusion des images produites autant par les photographes que par les adoptants et moyen d'expérimentation en ligne du projet et du paysage, permettant de circuler dans les images et de les comparer, de les situer sur une carte interactive, de participer à une communauté pour contribuer avec des commentaires, etc. Mais là encore, qu'il s'agisse de la formation à la reconduction, des marches commentées du paysage, de la gestion de la plateforme en ligne, le fonctionnement et la qualité des propositions dépendent des deux artistes, dont l'expertise photographique et paysagère est en réalité peu confrontée à celle d'acteurs extérieurs, d'une part, et dont la capacité d'action reste limitée, d'autre part. Plus largement, de nouveau, ceux-ci se trouvent confrontés à un biais inhérent à leur principe initial et voient se répercuter à leur échelle les difficultés rencontrées habituellement par les structures porteuses. Les mêmes questions se posent, mais de manière particulièrement sensible pour ces porteurs indépendants: quelle résonance du projet, quels moyens, quelle pérennité en l'absence d'institution relais, etc.? Justement, si la médiation locale, assurée par les photographes, mobilise et sensibilise notamment les adoptants, qu'en est-il des relations à d'autres interlocuteurs, telles que la Mission interministérielle métropole Aix-Marseille-Provence ? Celle-ci s'est emparée du corpus réalisé pour renforcer sa réflexion et son discours sur la métropole, mais ne s'est pas impliquée en amont du projet. Son usage des photographies lors des conférences 
métropolitaines et sur des supports de communication (en ligne) mérite d'être interrogé car il semble se situer à la fois entre réel usage des images, comme moyen de révélation des complexités du territoire et de sensibilisation des publics et des différents acteurs de la future métropole, et outil de marketing territorial par la caution artistique.

Figure 21. Coffret de cartes postales

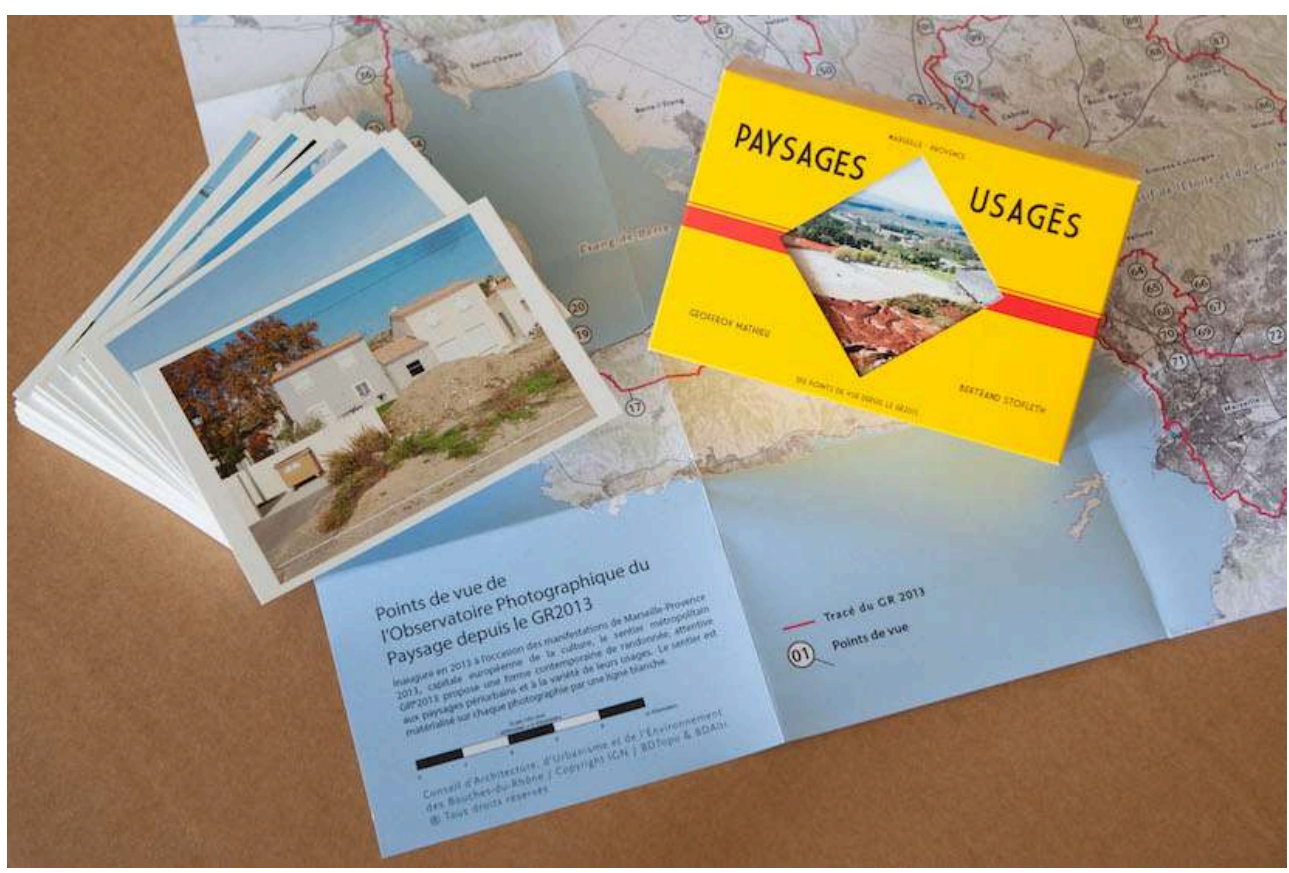

Sources: OPP depuis le GR2013. Bertrand Stofleth et Geoffroy Mathieu.

Figure 22. Vue d'exposition

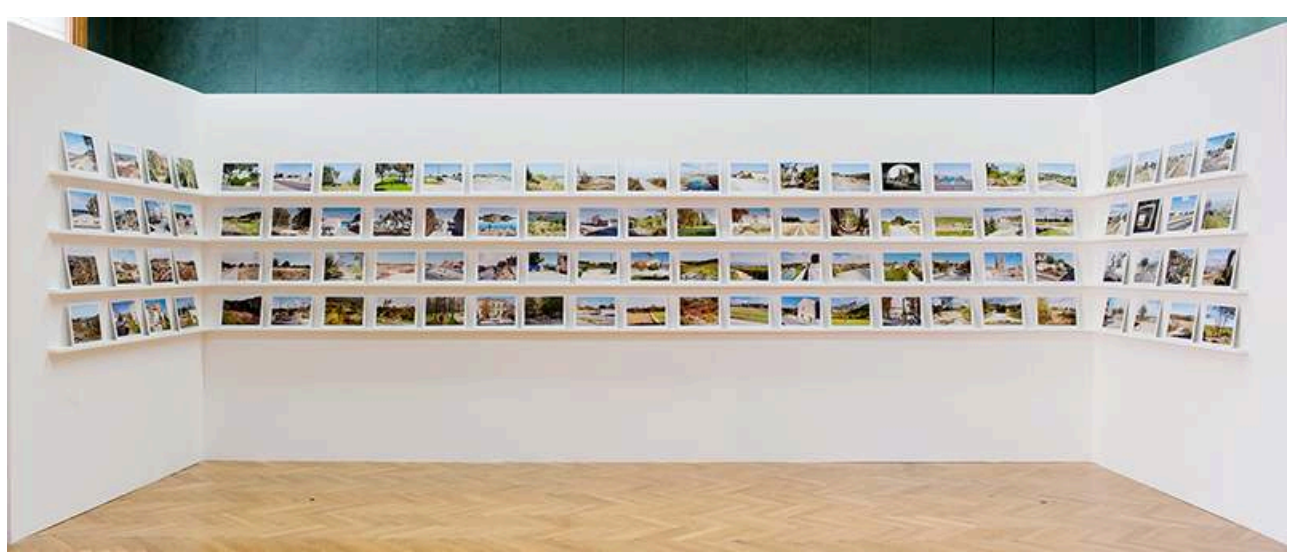

Sources : OPP depuis le GR2013. Bertrand Stofleth et Geoffroy Mathieu

39 C'est donc surtout à l'échelle locale que l'OPP, dispositif de mise en représentation et de médiation plurielle, devient moyen d'une expérience spatiale permettant l'implication et la responsabilisation citoyennes. Les Panoramistes fondent leur action sur une compréhension du paysage nourrie par la pensée d'Augustin Berque, qui l'envisage comme passage entre le matériel et le symbolique, le physique et le phénoménal, autant que comme forme de relations entre les individus et leur milieu. Ils partagent 
ainsi le fondement conceptuel de la réflexion sur les modalités et perspectives d'un paysage comme objet, enjeu et outil de médiation et comme «bien commun » (Sgard, 2010) et expérimentent d'une certaine manière, avec ces perspectives et écueils, une mise en œuvre des hypothèses de l'Observatoire photographique. Avec «L'OPP depuis le GR2013 ", ils proposent en effet un outil autant pratique que symbolique et des supports pour penser la trajectoire du paysage, pour débattre et se projeter sur le devenir du territoire, en imaginant par l'image des valeurs communes. Et pour faire ce portrait raisonné de la métropole à travers le paysage abordé comme vecteur du sentiment d'appartenance et de l'identité des territoires, les photographes souhaitent " privilégier l'approche ressentie des territoires » (Les Panoramistes, 2012) et mettre au cœur de leur protocole l'expérience, dans une sorte de mise en abyme documentaire. Il s'agit, en s'adossant au GR, de documenter l'expérience de la marche, celle de traversée des territoires métropolitains et celle, esthétique, des paysages. La démarche fait écho aux principes de l'esthétique environnementale, qui envisage l'esthétique au-delà de la pratique artistique, comme expérience implicative globale sollicitant tous les sens et permettant l'appréhension de l'environnement et la mise en relation de chacun au monde. L'OPP, en tant qu'invitation à la marche sur le GR et donc immersion dans des espaces au quotidien seulement traversés, en tant que proposition de représentation du paysage et objet de médiation plurielle, relève d'une tentative de montrer que l'esthétique et le sensible sont des dimensions de la relation entre la société et son environnement. Plus encore, parce qu'elles contribuent à rendre le monde habitable, elles peuvent être un moyen de développement de pistes pour une gestion et une politique du territoire, par le paysage, débattues collectivement (Brady, 2007).

\section{Du récit à « imagibilité » métropolitaine : vers le projet ?} démarche nous encourage à élargir nos hypothèses sur les statuts du paysage comme enjeu, ressource, outil, selon une acception aujourd'hui élargie et hybridée du projet de territoire, ainsi qu'à interroger le projet photographique selon une perspective différente, considérant le rôle structurant de l'imaginaire spatial par la mise en représentation et en récit.

«L'OPP depuis le GR2013 » est retranscription, mais peut également être envisagé comme un mode de production d'un territoire en mutation. En effet, l'espace, en tant que phénomène idéel et matériel, résultat d'un dialogue entre éléments physiques, pratiques, idées, pensées, récits, est nourri d'un imaginaire spatial constitué d'images, matérialisées par les représentations, médiatrices du rapport entre l'individu et le monde. Ce projet apparaît donc comme une pratique spatiale, consistant autant à expérimenter les espaces qu'à en formuler l'imaginaire par des propositions visuelles permettant de donner du sens, d'identifier et de s'identifier aux lieux. Il tente de réactualiser des représentations obsolètes des paysages de cette métropole en émergence, pour contribuer au sentiment d'appartenance territoriale en valorisant la dimension sensible, esthétique et affective du paysage. Plus fondamentalement, il contribue à la production spatiale, par le pouvoir performatif de l'image. En effet, la photographie "ne représente pas seulement ce qui lui serait un antécédent, mais participe au processus de constitution de nos espaces. Elle fait advenir le monde spatial dans un ordonnancement possible. Et cet espace figuré par l'iconographie n'est pas seulement une image, double mimétique d'un espace qui serait lui, "réel", mais aussi

Projets de paysage, 15 | 2016 
déjà de l'espace en propre, de l'espace en plus» (Lussault, 2007, p. 80). Pour cela, les auteurs se confrontent à la « crise figurative de l'urbain " (Lussault, 2007, p. 296.), au défi de la représentation d'une réalité dilatée, fragmentée, diluée, etc., dont l'imagibilité est difficilement saisissable et formulable. Le projet peut ainsi être observé comme support d'interrogation de cette notion conçue par Kevin Lynch, désignant la capacité des villes à développer chez les individus des images mentales claires et structurées des espaces, leur permettant de s'orienter, d'identifier et de s'identifier (Lynch, 1960). Comment passer aujourd'hui de la production de l'imagibilité de la ville à celle des espaces complexes de la métropole, et donc plus largement de l'espace habité par l'homme, urbain et non urbain?

Figure 23. La Busserine, Marseille, septembre 2012

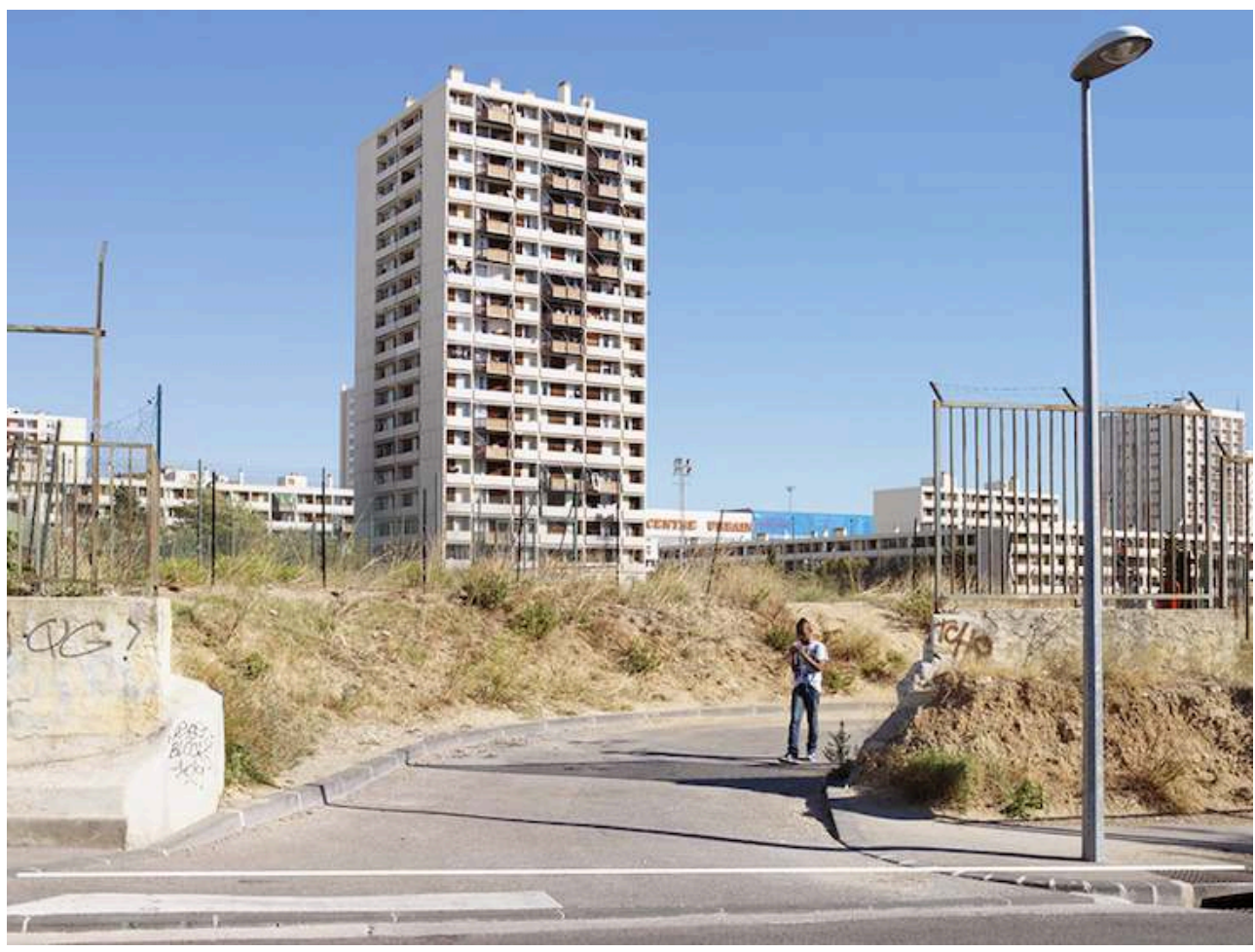

Les photographes tentent de saisir la diversité des espaces métropolitains et des différents modes d'occupation de la nature, dans un ensemble complexe à différentes échelles, où une imagerie a priori désirable peut être confrontée, parfois dans une même image, à la représentation de réalités urbaines difficilement identifiables.

Sources: OPP depuis le GR2013. Bertrand Stofleth et Geoffroy Mathieu 
Figure 24. Vallon Dol, Marseille, juin 2012.

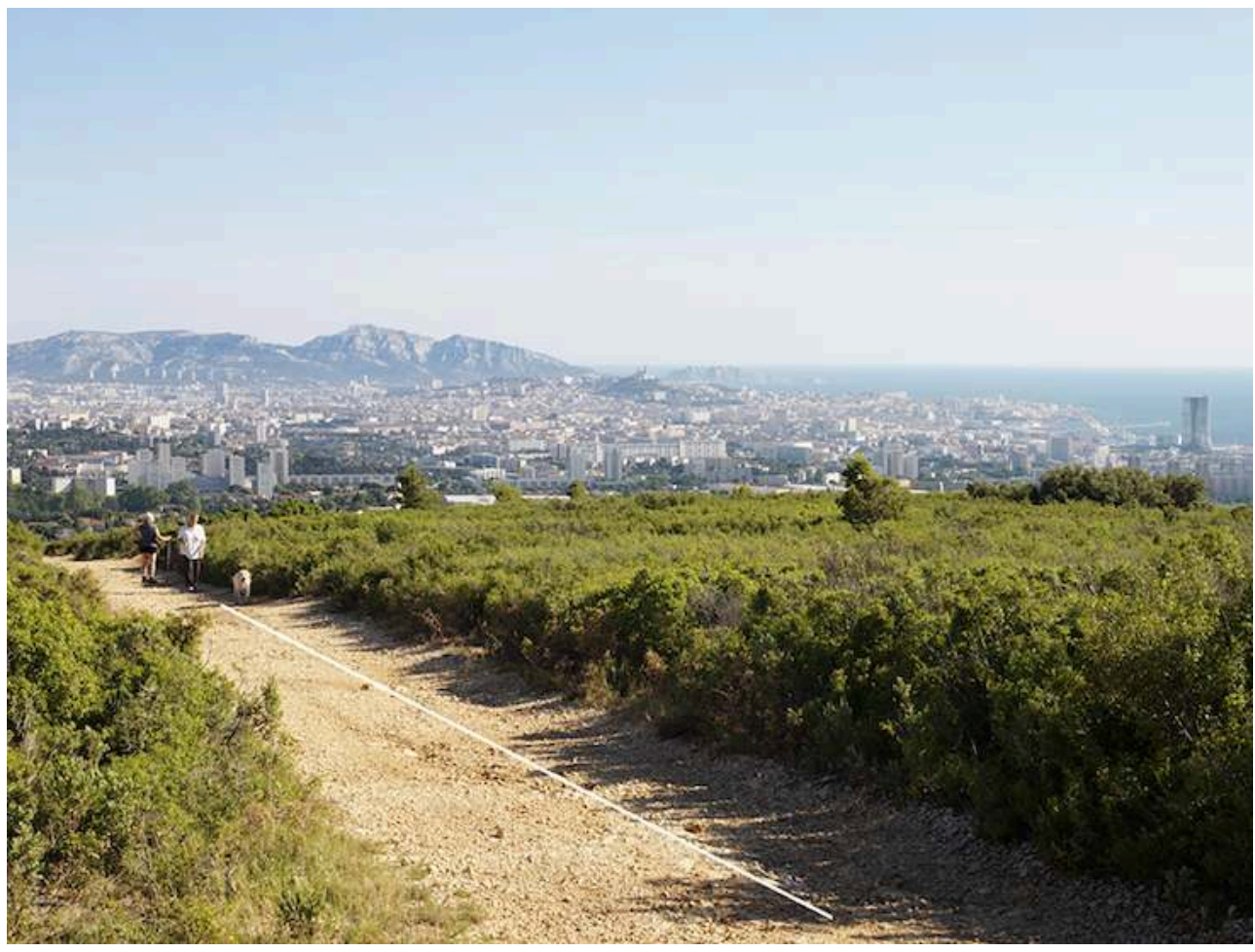

Sources : OPP depuis le GR2013. Bertrand Stofleth et Geoffroy Mathieu

Figure 25. Plage des Combattants, Port de Bouc, 2012

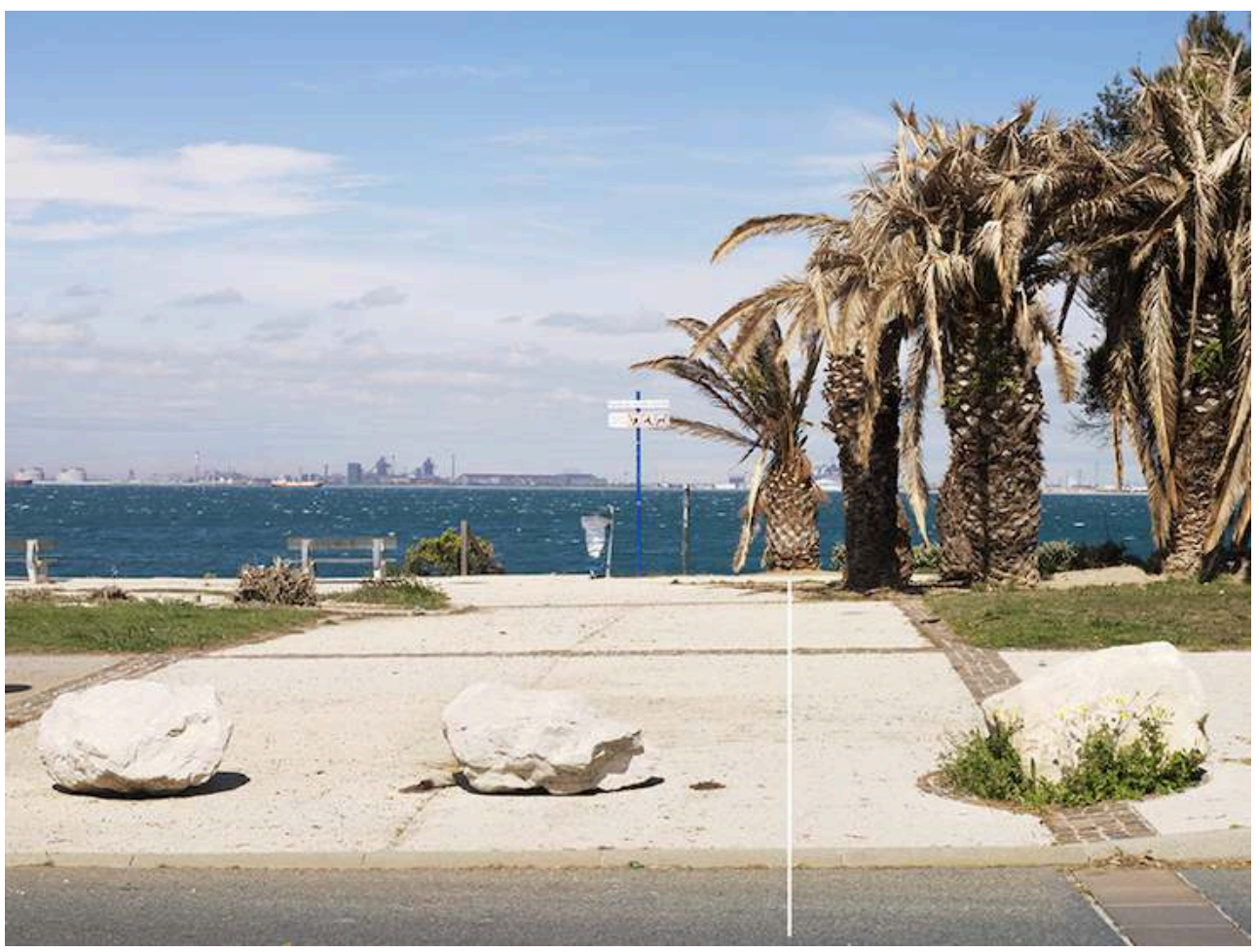

Sources : OPP depuis le GR2013. Bertrand Stofleth et Geoffroy Mathieu 


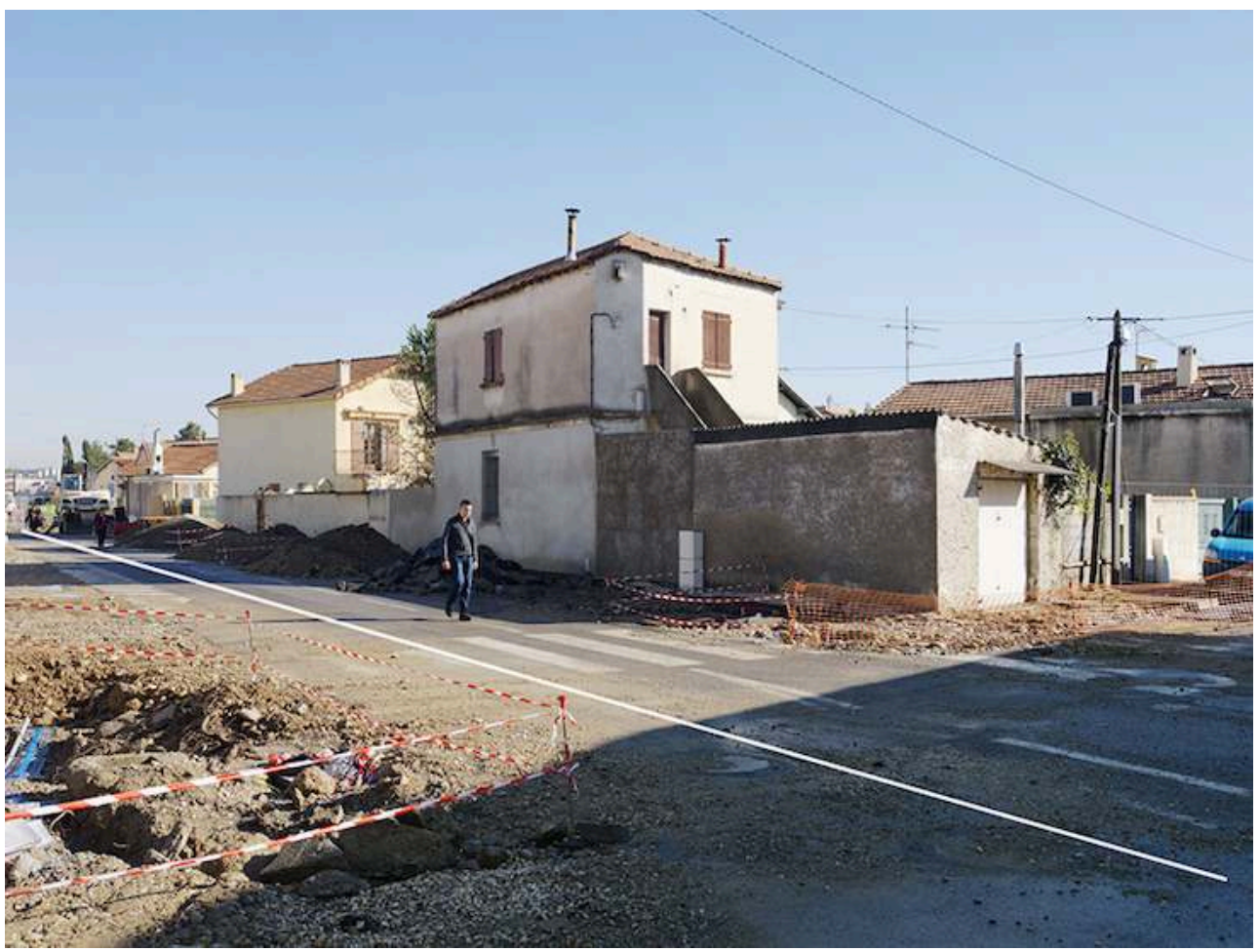

Sources : OPP depuis le GR2013. Bertrand Stofleth et Geoffroy Mathieu

L'OPP constitue le volet d'une infrastructure plus large, articulée autour du GR2013 qui, pensé comme un projet politique, met en œuvre une dynamique d'élargissement et de complexification des dispositifs de projet d'aménagement à partir de l'hypothèse du vécu comme support de projet. Proposant de (se) rassembler sinon pour faire consensus, du moins pour faire converger des regards, il tente de construire une histoire collective de la métropole. Le GR2013 et ses images misent donc sur le récit et ses figures dans leur capacité à décrire une réalité existante, proposer un état du monde, mais également à générer un récit autour duquel se rassembler et à partir duquel projeter (Lussault, 1998). En effet, tout projet de territoire est préalablement un récit et c'est par le truchement de l'image, performative, que les instances politiques formulent et s'approprient la territorialité, la configurent physiquement et idéologiquement. Depuis 2014, le Bureau des guides en charge du GR2013 s'approprie ces processus de fabrication territoriale pour les réinventer et les déplacer vers la société civile. Et Les Panoramistes procèdent de même en détournant la méthode de l'observatoire. Ils réévaluent le rôle des acteurs (institutions et citoyens), repensent les différentes expertises en insistant sur la dimension empirique ; et surtout, mettent au cœur de leur action l'expérience du paysage, envisagé comme vecteur de médiation plurielle. Ils produisent alors une expérimentation symptomatique du renouvellement des modèles théoriques, pratiques et de représentation $d u$ et pour le paysage, remettant au cœur des enjeux l'imaginaire spatial, par le biais de la pratique artistique, pour en faire un outil de projection et de projet.

«L'OPP depuis le GR2013 » met en œuvre une photographie comme pratique spatiale, mais également territoriale, puisqu'il s'agit de représenter les réalités et problématiques matérielles, sociales, politiques, à l'œuvre en l'occurrence dans le 
projet métropolitain Aix-Marseille-Provence et son territoire fortement marqué par la périurbanité. En appliquant la méthode d'observation photographique des paysages au champ de l'urbain, les photographes déplacent les possibilités du programme, qu'ils font fonctionner au niveau culturel, la dimension symbolique d'une culture en l'occurrence métropolitaine étant alors envisagée comme vecteur de réalisation. Tout en proposant des représentations actualisées des paysages et une imagibilité urbaine contemporaine, ils agissent au niveau pratique et opérationnel en mettant le processus en interaction avec l'espace social, les publics et les territoires. En cela, cette démarche, fruit du «tournant spatial » de l'art, rejoint le concept de « ré-outillage artistique des territoires" développé par le Pôle des arts urbains (Polau, 2015) pour désigner une dynamique d'engagement de la création dans la sphère de l'aménagement: qu'elle produise des connaissances utiles à la conception ou accompagne les transformations d'un territoire, la création est envisagée d'abord selon ses processus, mettant en avant les dispositifs d'invention et de médiation de l'art.

«L'OPP depuis le GR2013 », pensé comme mise en abyme de dispositifs d'expériences, de regards et de représentations dans le contexte d'une infrastructure de projet plus large, fait émerger images et modèles de paysages métropolitains et propose de nouveaux modèles d'action pour la méthode initiée par l'OPNP. Plus largement, le renouvellement de la méthode, porté notamment par une reconfiguration du rôle des acteurs, une compréhension élargie des champs d'action de l'art et de "ce que le territoire fait à la création » ainsi que «ce que la création fait au territoire » (Polau, 2015), démontre une transversalité croissante dans le projet d'aménagement du territoire. Enfin, Geoffroy Mathieu et Bertrand Stofleth, par ce manifeste en action, affirment le caractère politique du statut de faiseur d'images et rappellent la dimension idéologique propre à toute observation: ils exploitent un facteur de la méthode de l'observation photographique qui avait jusqu'à présent été sous-évalué et participent à une réflexion propre à la photographie documentaire, qui interroge "l'activité artistique comme laboratoire de production de savoirs" selon une "éthique de la représentation. »(Beausse, 2012, p. 272).

\section{BIBLIOGRAPHIE}

Beausse, P., « Documents pour une information alternative », Les Rencontres Arles photographie 2012. Une école française, Arles, Actes Sud, 2012, p. 272-280.

Berque, A., Médiance, de milieux en paysages, Paris, Belin, coll. « Reclus géographiques », 2000, $164 \mathrm{p}$.

Brady, E., « Vers une véritable esthétique de l'environnement : élimination des frontières et des oppositions dans l'expérience esthétique du paysage », Cosmopolitiques. Esthétique et espaces publics, $\mathrm{n}^{\mathrm{o}}$ 15, 2007, Rennes, Éditions Apogée, p. 65-76. 
Bonn, S., « Le projet comme dispositif de vision du paysage, » Projets de paysage, $\mathrm{n}^{\circ} 1$, décembre 2008, URL : https://www.projetsdepaysage.fr/fr/ le_projet_comme_dispositif_de_vision_du_paysage

Briffaud, S., «Le paysage, le regard et le temps. Enquête historique et géographique sur l'empaysagement des espaces des sociétés », mémoire pour l'HDR, résumé des travaux, université Bordeaux Montaigne, 2013, 5 p.

Claass, A., Le Réel de la photographie : médiations sur l'image, Trézélan, Filigranes, 2013, 300 p.

Davodeau, H., Toublanc, M., « Le paysage-outil, les outils du paysage. Principes et méthodes de la médiation paysagère ", congrès « Coconstruction ou construction en commun d'objectifs collectifs », Montpellier, octobre 2010.

DGALN/DHUP, « Observatoires photographiques du paysage « locaux », recensement et typologie », décembre 2015, $27 \mathrm{p}$.

Donadieu P., « Le paysage, un paradigme de médiation entre l'espace et la société ? », Économie rurale, $\mathrm{n}^{\circ}$ 297-298, 2007, p. 5-9.

Donadieu P., « De la production de l'espace à celle du rapport social à l'espace. Le double sens du paysage ", «Itinéraires croisés ", actes des rencontres de l'observatoire photographique du paysage, les 24 et 25 septembre 1999 à Rochefort, ministère de l'Aménagement du territoire et de l'Environnement/ville de Rochefort/région Poitou-Charentes, 1999, 29-38 p

Dubois, C., « Le paysage, enjeu et instrument de l'aménagement du territoire », Biotechnologie, Agronomie, Société et Environnement, vol. 13, nº 2, 2009, p. 309-316.

Fédération des Parcs naturels régionaux de France, « Étude sur les Observatoires photographiques du paysage dans les parcs naturels régionaux », mars 2015, $96 \mathrm{p}$.

Jakob, M., Le Paysage, Gollion, InFolio, coll. « Archigraphy », 2008, 192 p.

Jakob, M, Paysage et temps, Gollion, InFolio, coll. « Archigraphy », 2007, 140 p.

Lenclud, G., «L'ethnologie et le paysage. Questions sans réponse », dans Voisenat, C., Paysages au pluriel : pour une approche ethnologique des paysages, Paris, Éditions de la Maison des sciences de l'homme, 1995, p. 2-17.

Luginbühl, Y., « La demande sociale de paysage. Rapport devant le Conseil national du paysage », 28 mai 2001, 19 p.

Lussault, M., L'Homme spatial, la construction sociale de l'espace humain, Paris, Éditions du Seuil, coll. «La Couleur des Idées », 2007, 363 p.

Lussault, M., «Images (de la ville) et politique territoriale », Revue géographique de Lyon, vol. 73, $\mathrm{n}^{\circ}$ 1,1998, p. 45-53.

Lynch, K., L'Image de la ville (1960), Paris, Dunod, 1999, 221 p.

Les Panoramistes/Mathieu, G. et Stofleth, B., « Points de Vie. Paysages usagés, Observatoire photographique depuis le GR2013, 2012-2022 », dossier de presse, 2012.

Marcel, O., « Voir le paysage (d')aujourd'hui », « Itinéraires croisés », actes des rencontres de l'Observatoire photographique du paysage, les 24 et 25 septembre 1999 à Rochefort, ministère de l'Aménagement du territoire et de l'Environnement/ville de Rochefort/région Poitou-Charentes, 1999, 111-133 p. 
Ministère de l'Écologie, de l'Énergie, du Développement durable et de l'Aménagement du territoire, «Itinéraires photographiques. Méthode de l'Observatoire photographique du paysage ", Paris, 2008, 74 p.

Mollie-Stefulesco Caroline, "L'Observatoire photographique du paysage », Séquences Paysages. Revue de l'Observatoire photographique du paysage, Paris, ministère de l'Environnement/Hazan, $\mathrm{n}^{\circ} 1$, 1997, p. 4-10.

Mollie-Stefulesco Caroline, " Huit ans d'Observatoire ", Séquences Paysages. Revue de l'Observatoire photographique du paysage, Paris, ministère de l'Environnement/Arp Éditions, nº 2, 2000, p. 4-6.

Moiroux, F., « Le GR2013, un état des lieux sensible de la métropole phocéenne », AMC, $\mathrm{n}^{\circ} 225$, juin-juillet 2013.

Piveteau V. «La photographie de paysage, un outil de médiation pour les territoires », «Itinéraires croisés », actes des rencontres de l'observatoire photographique du paysage, les 24 et 25 septembre 1999 à Rochefort, ministère de l'Aménagement du territoire et de l'Environnement/ville de Rochefort/région Poitou-Charentes, 1999, 69-77 p.

PNTH, « Mise en œuvre et retour d'expériences. Éléments méthodologiques », 2014, 40 p.

Polau, « Plan-guide, “arts et aménagement des territoires”, étude nationale pour le ministère de la Culture et de la Communication », mai 2015, np.

Quesney, D. « Méthode photographique », Ponts et Chaussées magazine, $\mathrm{n}^{\circ}$ 4, 2008, p. 64-70

Quesney D., «L'intuition des photographes », Séquences Paysages. Revue de l'Observatoire photographique du paysage, Paris, ministère de l'Environnement/Hazan, n 1, 1997, p. 22-26.

Ristelhueber Véronique, «Les séries dans les fonds photographiques ", Séquences Paysages. Revue de l'Observatoire photographique du paysage, Paris, ministère de l'Environnement/Hazan, $\mathrm{n}^{\circ} 1,1997$, p. 86-91.

Roger, A. (dir.), La Théorie du paysage en France, 1974-1994 (1995), Seyssel, Champ Vallon, 2009, $463 \mathrm{p}$.

Rouillé, A., La Photographie, entre document et art contemporain, Paris, Gallimard, coll. « Folio essais », 2005, $704 \mathrm{p}$.

Sgard, A., « Le paysage dans l'action publique : du patrimoine au bien commun », Développement durable et territoires [en ligne], vol. 1, $\mathrm{n}^{\circ}$ 2, septembre 2010, URL : http:// developpementdurable.revues.org/8565.

Sgard, A., Fortin M.-J, et Peyrache-Gadeau, V., « Le paysage en politique », Développement durable et territoires [En ligne], vol. 1, $\mathrm{n}^{\circ}$ 2, septembre 2010, URL : http:// developpementdurable.revues.org/8522.

Volvey, A., « Entre l'art et la géographie, une question (d')esthétique », Belgeo [En ligne], nº 3, septembre 2014, URL : http://belgeo.revues.org/13258.

\section{NOTES}

1. Adoptée le 20 octobre 2000, à Florence par 29 États-membres.

2. Entretien, 25 novembre 2015.

3. Titre du colloque dirigé en 1981 par François Dagognet.

4. C'est l'auteur qui souligne. 
5. En 1995 sera publié, sous la direction d'Alain Roger, l'ouvrage La Théorie du paysage en France, 1974-1994, réunissant, notamment ces auteurs.

6. C'est l'auteur qui souligne.

7. C'est l'auteur qui souligne.

8. C'est l'auteur qui souligne.

9. Et ensuite, les OPP.

10. Il fait d'ailleurs appel à une photographe développant une pratique artistique, Édith Roux.

11. Le terme " analyse ", tout comme celui de "scientifique » et «technique ", appliqué à une démarche d'observation photographique, est par ailleurs à questionner.

12. Notons d'ailleurs la surprenante formule de l'introduction de la méthode de 2008 : «Il est nécessaire de développer la connaissance des quelques 2000 paysages qui composent notre pays. » 13. Le statut de l'artiste est alors exploité comme labellisation culturelle, bien souvent sous une forme relevant d'une compréhension passéiste de la pratique artistique.

14. Capitale européenne de la culture.

15. Concept développé par le Bureau des guides, association en charge du GR2013 depuis 2014, comme outil de réflexion et d'action territoriale.

16. Journée "Transformations », journée d'échange relative aux 25 ans de la démarche OPP, le 21 juin 2016.

17. Ces thématiques sont détaillées dans l'entretien cité précédemment.

18. Ibid.

19. Plutôt que de "sortir du milieu de l'art ", ils souhaitent exister dans plusieurs milieux, la sphère artistique restant leur espace de production et de légitimation. De plus, c'est surtout par le Cnap que le projet est financé.

20. Trente images choisies en comité de pilotage étant destinées à intégrer les collections du Cnap.

21. Les photographes reviennent sur leur démarche dans l'entretien.

22. En 2013, six rendez-vous ont eu lieu sur différentes étapes du GR.

\section{RÉSUMÉS}

C'est dans un contexte de visibilité extrême du paysage comme discours, texte et image, que les initiatives d'observation photographique du paysage connaissent une vraie dynamique sur le territoire français. Ces démarches, héritières du programme de l'observatoire photographique national du paysage (OPNP), reprennent et transforment une "méthode » qui a été érigée en «modèle ». La multiplication des Observatoires photographiques du paysage (OPP) est pourtant paradoxale. En effet, pourquoi la démarche connaît-elle un tel succès, compte tenu des difficultés et des critiques énoncées de manière récurrente par les structures porteuses, en matière de mise en œuvre et d'exploitation de l'« outil »? Cet article vise à éclairer les raisons de ce paradoxe en proposant d'abord un retour sur le contexte de l'expérimentation qu'a constitué la création de l'OPNP. Il s'agit également d'opérer un retour critique sur les fondements théoriques et conceptuels (même non formulés) portés par un programme à la dimension rhétorique importante, pour les confronter aux dispositifs de mise en œuvre. Une question se pose, permettant d'envisager une réévaluation de la démarche, après 25 ans d'existence : "l'héritage OPNP » serait-il la reconduction à la fois des apports d'un programme expérimental qu'il 
convient aujourd'hui de reconsidérer, mais aussi de ses ambivalences, de ses lacunes, voire de ses contresens? Enfin, une initiative particulière offre un support de réflexion sur les potentiels et perspectives de réactualisation de l'héritage et les possibilités de l'observation photographique appliquée aux territoires. En effet, «Paysages usagés, Observatoire photographique du paysage depuis le GR2013 », né d'une initiative de relecture critique de la méthode par les photographes Bertrand Stofleth et Geoffroy Mathieu, propose une réévaluation des perspectives de l'observation photographique, tournée vers l'expérience esthétique du paysage comme médiation plurielle et mettant en œuvre, par le dispositif produit, le projet artistique au cœur des réflexions territoriales.

In the context of the landscape's extreme prominence as a discourse, composition, and image, initiatives for the photographic observation of the landscape are developing in France. These approaches inherited from the Observatoire photographique national du paysage (OPNP), take inspiration from a "method" which has been put forward as a "model". The creation of so many Photographic Observatories of the Landscape presents a paradox. Why is this initiative such a success, given the difficulties encountered and the recurring criticism expressed by leading organisations concerning the implementation of this "tool"? This article seeks to shed light on the reasons behind this paradox by looking back at the context that gave rise to the OPNP experiment. The article also takes a critical look at the founding theoretical and conceptual principles (even those not formulated) which inspired a programme with such a strong rhetorical dimension and compares these principles with the systems implemented. A question arises concerning the possible reassessment of this approach after 25 years: Might not the "the OPNP heritage" be the continuation of an experimental programme the contributions, ambivalences, shortcomings and contradictions of which need to be re-examined today? Lastly, a specific initiative offers an interesting case study on the potential and perspectives for a recontextualisation of the heritage and the potential for applying photographic observation to landscapes. "Paysages usagés, Observatoire photographique du paysage depuis le GR2013" ("Used Landscapes, A Photographic Observatory of the Landscape from the GR2013 Trail"), which was born of the initiative of a critical reinterpretation of the method by photographers Bertrand Stofleth and Geoffroy Mathieu, proposes a re-evaluation of the perspectives of photographic observation focusing on the aesthetic experience of the landscape as a plural mediation placing an artistic project at the heart of territorial planning.

\section{INDEX}

Mots-clés : représentation, paysage, photographie, médiations, récit, dispositif

Keywords : representation, landscape, photography, mediations, narrative, system

\section{AUTEUR}

\section{FRÉDÉRIQUE MOCQUET}

Architecte, diplômée d'État, elle mène depuis 2013 un doctorat au sein du laboratoire Architecture, Culture, Société [ACS, UMR AUSser 3329] à l'École nationale supérieure d'architecture Paris-Malaquais. Sa recherche, intitulée « Paysages photographiques : représentations, aménagement du territoire et prospective », explore la dimension performative des représentations d'un point de vue symbolique et pratique et envisage notamment l'aptitude d'une photographie dite « de paysage » à contribuer à un imaginaire collectif autant qu'à devenir 
objet et outil d'analyse.

fmocquet[at]gmail[dot]com 